\title{
THE FIBONACCI UNIMODAL MAP
}

\author{
MIKHAIL LYUBICH AND JOHN MILNOR \\ Dedicated to Yuri Lyubich on the occasion of his sixtieth birthday
}

\section{INTRODUCTION}

The Fibonacci recurrence of the critical orbit appeared in the work of Branner and Hubbard on complex cubic polynomials [BH, §12] and in Yoccoz's work [Y1, Y2] on quadratic ones, as the "worst" pattern of recurrence. On the other hand, a real quadratic Fibonacci map was suggested by Hofbauer and Keller [HK] as a possible candidate for a map having a "wild" attractor (that is, a set $A$ which is the $\omega$-limit set for Lebesgue almost every orbit but is strictly smaller than the $\omega$-limit set for a generic orbit). The $\omega$-limit set of the critical orbit in $[\mathrm{HK}]$ possesses all known topological properties of wild attractors (compare [BL2]). In fact, we will see below that the quadratic Fibonacci map does not have a wild attractor; however, the corresponding question for a map with a degenerate critical point remains open.

Actually, the first indication of the Fibonacci map appeared in the numerical work of Tsuda [T], related to the Belousova-Zhabotinskii reaction, and also in numerical work of Shibayama [Sh] (more precisely, they studied the sequence of "Fibonacci bifurcations" creating the Fibonacci map).

This paper will study topological, geometrical, and measure-theoretical properties of the real Fibonacci map. Our goal was to figure out if this type of recurrence really gives any pathological examples and to compare it with the infinitely renormalizable patterns of recurrence studied by Sullivan [S]. It turns out that the situation can be understood completely and is of quite regular nature. In particular, any Fibonacci map (with negative Schwarzian and nondegenerate critical point) has an absolutely continuous invariant measure (so, we deal with a "regular" type of chaotic dynamics). It turns out also that geometrical properties of the closure of the critical orbit are quite different from those of the Feigenbaum map: its Hausdorff dimension is equal to zero and its geometry is not rigid but depends on one parameter.

Branner and Hubbard introduce the concept of a tableau in order to describe recurrence of critical orbits. Their "Fibonacci tableau" is a basic example, which corresponds to one particularly close and regular pattern of recurrence. If a complex quadratic map $z \mapsto z^{2}+c$ realizes this Fibonacci tableau, then the

Received by the editors February 12, 1992.

1991 Mathematics Subject Classification. Primary 58F03, 58F23.

The work of the first author was supported in part by NSF and by the Sloan Foundation. 
orbit

$$
0=z_{0} \mapsto z_{1} \mapsto z_{2} \mapsto \cdots
$$

of the critical point returns closer to zero (in a certain invariant sense) after each Fibonacci number of iterations. In the real case, it follows that

$$
\left|z_{1}\right|>\left|z_{2}\right|>\left|z_{3}\right|>\left|z_{5}\right|>\left|z_{8}\right|>\left|z_{13}\right|>\cdots .
$$

In $\S 2$ we will prove that a real quadratic map is uniquely defined by the last property; more precisely, we prove the following. We denote the Fibonacci numbers by

$$
u(1)=1, u(2)=2, \ldots \quad \text { with } u(n+1)=u(n)+u(n-1) .
$$

Theorem 1.1. There is one and only one real quadratic map of the form $f_{c}(x)=$ $x^{2}+c$ with the property that the critical orbit $0=x_{0} \mapsto x_{1} \mapsto \cdots$ has closest recurrence at the Fibonacci values, so that $\left|x_{1}\right|>\left|x_{2}\right|>\left|x_{3}\right|>\left|x_{5}\right|>\cdots$, with $\left|x_{3}\right|<\left|x_{4}\right|$. The kneading invariant for this uniquely defined map $f_{c}$ can be described by the conditions that

$$
\begin{aligned}
& x_{u(n)}<0 \quad \text { for } n \equiv 0,1 \bmod 4, \\
& x_{u(n)}>0 \quad \text { for } n \equiv 2,3 \bmod 4,
\end{aligned}
$$

and that

$$
\operatorname{sgn}\left(x_{i}\right)=\operatorname{sgn}\left(x_{i-u(n)}\right) \quad \text { for } u(n)<i<u(n+1) .
$$

In fact, numerical computation shows that

$$
c=-1.8705286321646448888906 \ldots
$$

The associated topological entropy is $h=\log 1.7292119317 \ldots$.

For a fairly general unimodal map $f$ with this same kneading data, we prove the following. Let $\mathscr{O}=\left\{x_{0}, x_{1}, \ldots\right\} \subset \mathbf{R}$ be the critical orbit.

Theorem 1.2. If $f$ is $C^{2}$-smooth with nonflat critical point, and with kneading data as above, then

(1) The closure $\overline{\mathscr{O}}$ of the critical orbit is a Cantor set, with the $x_{i}, i \geq 1$, as the end points of the complementary intervals.

(2) The map $f$ from $\overline{\mathscr{O}}$ onto itself is one-to-one except that the critical point has two preimages. This map $f \mid \overline{\mathscr{O}}$ is minimal and is uniquely ergodic with entropy zero. It is semiconjugate to the golden rotation

$$
t \mapsto t-(\sqrt{5}-1) / 2(\bmod 1)
$$

of the circle $\mathbf{R} / \mathbf{Z}$.

The proof, in $\S 3$, will give an explicit description of the ordering of this critical orbit closure. It will also show that it is canonically homeomorphic to the set of all infinite sequences $\left(a_{1}, a_{2}, \ldots\right)$ of zeros and ones with no two consecutive ones, or to the set of all finite or infinite "Fibonacci sums". (Compare Definition 2.2 and Lemma 3.3.) 
Theorem 1.3. If $f$ is $C^{2}$-smooth with nondegenerate critical point, then

(1) The ratio of $x_{u(n)}$ to $x_{u(n-1)}$ decreases exponentially, with

$$
\lambda_{n} \equiv\left|x_{u(n)}\right| /\left|x_{u(n-1)}\right| \sim a / 2^{n / 3} \text { as } n \rightarrow \infty
$$

for some constant $a>0$.

(2) The critical orbit closure $\overline{\mathscr{O}}$ has Hausdorff dimension zero, and the Liapunov exponent at the critical value is equal to zero.

(3) Any two Fibonacci maps with the same parameter a are smoothly conjugate on $\overline{\mathscr{O}}$.

(4) If the Schwarzian derivative is negative, then $f$ has a unique absolutely continuous invariant measure, with support equal to the entire closed interval $\left[x_{1}, x_{2}\right]$ and with positive entropy.

Remark 1.1. Uniqueness and other properties of an absolutely continuous invariant measure hold automatically (see [BL2]). We will derive existence from the Nowicki-van Strien "series" condition [NvS].

Remark 1.2. Unlike the Feigenbaum map, the geometry of $\overline{\mathscr{O}}$ goes down to zero and is not rigid but depends on the parameter $a$. (We can effectively vary this parameter.)

Remark 1.3. It is essential here that the critical point be nondegenerate $\left(f^{\prime \prime}(0) \neq 0\right)$. For example, a Fibonacci map of the form $f(x)=x^{4}+c$ has completely different behavior, with bounded geometry and perhaps with no absolutely continuous invariant measure. A computer experiment by Lyubich and Tangerman suggests that the corresponding map $x \mapsto x^{6}+c$ has the Cantor set $\omega(0)$ as "wild" attractor.

Let us describe the structure of the proof of the last theorem, which is somewhat complicated. In $\S 4$ we get some a priori bounds on the ratios $\lambda_{n}$. In $\S 5$ we prove Theorem 1.3 assuming that $\inf \lambda_{n}=0$. In order to verify this assumption we introduce in $\S 6$ an appropriate notion of renormalization so that infinitely renormalizable maps are exactly Fibonacci ones. Applying Sullivan's ideas [S] to our case we prove that if geometry of $\overline{\mathscr{O}}$ is bounded from below, then there is a sequence of renormalizations converging to a map which can be analytically continued in a quite big domain of the complex plane.

In $\S 7$ we discuss polynomial-like maps, in an appropriate generalized sense. A version of the Douady-Hubbard theorem is valid in this situation: any cubiclike map is quasi-conformally conjugate to a cubic polynomial with one escaping critical point. It follows that all real cubic-like Fibonacci maps are quasisymmetrically conjugate. So, any example of a cubic-like Fibonacci map with unbounded geometry shows that all of them have unbounded geometry. Finally, we renormalize a quadratic-like Fibonacci map into a cubic-like one, which completes the proof for the polynomial-like case.

In the last $\S 8$ we show that the limits of the renormalizations of a smooth Fibonacci map are actually polynomial-like, which completes the proof of Theorem 1.3.

Remark 1.4. The Fibonacci recurrence is a well-known phenomenon for monotone maps of the circle with golden rotation number. The scaling laws in this 
situation were studied by Herman (at least implicitly), by Swiatek [Sw1] (smooth homeomorphisms with critical points), and by Tangerman and Veerman [TV] (maps with flat spots). In the two former cases one has bounded geometry, in the latter the geometry goes down to zero in the similar manner as in our example. Such circle maps are explicitly related to certain unimodal maps of the interval which are different from ours but also have a sort of Fibonacci recurrence; see [PTT].

The notation $f^{n}$ will always be used for the $n$-fold iterate of $f$.

\section{KNEADING}

Let $f: I \rightarrow I$ be a unimodal map with minimum at $x=0$. As usual, let $0=x_{0} \mapsto x_{1} \mapsto \cdots$ be the critical orbit, and let

$$
u(1)=1, \quad u(2)=2, \quad u(3)=3, \quad u(4)=5, \quad \ldots
$$

be the Fibonacci numbers. In order to avoid the hypothesis that $f$ is an even function, we will use the notation $x \mapsto x^{\prime}$ for the order-reversing involution, defined on some suitable subinterval of $I$, which satisfies $f\left(x^{\prime}\right)=f(x)$. Let $\|x\|$ be the larger of $x$ and $x^{\prime}$.

Definition 2.1. We will say that $f$ is a Fibonacci map if $\left\|x_{u(n)}\right\|>\left\|x_{u(n+1)}\right\|$ for $n \geq 1$, so that

$$
\left\|x_{1}\right\|>\left\|x_{2}\right\|>\left\|x_{3}\right\|>\left\|x_{5}\right\|>\left\|x_{8}\right\|>\left\|x_{13}\right\|>\cdots,
$$

and if $\left\|x_{3}\right\|<\left\|x_{4}\right\|$.

Lemma 2.1. The map $f$ is a Fibonacci map if and only if the signs of the successive images $x_{i}$ are given by

$$
\operatorname{sgn}\left(x_{j}\right)=\operatorname{sgn}\left(x_{j-u(n)}\right) \quad \text { for } u(n)<j<u(n+1)
$$

with

$$
\operatorname{sgn}\left(x_{u(n)}\right)=(-1)^{(n+1)(n+2) / 2}
$$

Remark 2.1. The condition $\left\|x_{3}\right\|<\left\|x_{4}\right\|$ may not be dropped. For example, the pattern

$$
x_{1}<x_{9}<x_{6}<x_{8}<x_{13}<0<x_{5}<x_{7}<x_{4}<x_{3}<x_{10}<x_{2}
$$

is compatible with (2.1).

Remark 2.2. We can describe these conditions in a different language as follows. If we assume that $x_{1}<0<x_{2}$, then Conditions (2.2) and (2.3) are completely equivalent to the statement that the interval between 0 and $x_{u(n)}$ is mapped homeomorphically by the iterate $f^{\circ i}$ for $0 \leq i \leq u(n-1)$ but is not mapped homeomorphically by $f^{\circ u(n-1)+1}$. The condition that some large iterate of $f$ restricted to an interval $[a, b]$ is a homeomorphism is an invariant way of specifying that $a$ is very close to $b$. Thus Lemma 2.1 can be thought of as giving an invariant description of just how close $x_{u(n)}$ is to the critical point.

Remark 2.3. The Branner-Hubbard description of $f$ would be rather different. Following Yoccoz, they cut the interval not at the critical point, but rather at the 
interior fixed point $\alpha<0$. In terms of the resulting partition of the interval, the appropriate description of the critical orbit is that the two images $x_{i}$ and $x_{i+u(n)}$ lie on the same side of $\alpha$ for $i<u(n+1)-2$ but on opposite sides of $\alpha$ for $i=u(n+1)-2$.

Proof of Lemma 2.1. If (2.2) and (2.3) are satisfied, then according to Remark 2.2, we see that the successive images $x_{u(n)}$ are closer and closer to zero. Since $f\left[x_{1}, x_{4}\right] \not \supset x_{4}$, it follows that $\left\|x_{4}\right\|>\left\|x_{3}\right\|$. Hence $f$ is a Fibonacci map. Conversely, the proof that every Fibonacci map satisfies (2.2) and (2.3) will be by induction on $n$, using the following induction hypothesis.

Hypothesis $\mathbf{H}_{n}$. For $i$ in the range $0<i<u(n)$ with $i \neq u(n-1)$, the points $x_{i}$ have sign as specified in conditions (2.2) and (2.3), and furthermore $\left\|x_{i}\right\|>\left\|x_{u(n-1)}\right\|$.

The following elementary observation will be used over and over. For any unimodal map with minimum at $x_{0}=0$

$$
\text { if }\left\|x_{p}\right\|<\left\|x_{q}\right\| \text { then } x_{p+1}<x_{q+1} \text {. }
$$

To start the induction, we must show that every Fibonacci map satisfies $\mathbf{H}_{4}$. Since $\left\|x_{1}\right\|>\left\|x_{2}\right\|>\left\|x_{3}\right\|$ and $\left\|x_{4}\right\|>\left\|x_{3}\right\|$ by definition, we need only show that $x_{1}, x_{4}<0<x_{2}$. Note first that the $\left\|x_{i}\right\|$ must all be distinct. For otherwise the critical orbit would have only finitely many distinct elements. If $0<x_{1}$ then we see inductively that $0<x_{1}<x_{2}<\cdots$, which contradicts our hypothesis. Similarly, if $x_{2}<0$ and hence $x_{1}<x_{2}<0$, then we see inductively that

$$
x_{1}<x_{3}<x_{5}<\cdots<x_{6}<x_{4}<x_{2}<0,
$$

which contradicts our hypothesis. Finally, let us show that $x_{4}<0$. Observe that $f(y)<y$ for $y \in\left[0, x_{2}\right]$. Thus, if $x_{3}>0$ then $x_{4}<x_{3}$ and hence $x_{4}<0$. If $x_{3}<0$ but $x_{4}>0$ then $x_{5}=f x_{4}<f x_{2}=x_{3}$ contradicting our hypothesis. This proves $\mathbf{H}_{4}$.

We will show that $\mathbf{H}_{n} \Rightarrow \mathbf{H}_{n+1}$ for $n \geq 4$. Since $0<\left\|x_{u(n)}\right\|<\left\|x_{u(n-1)}\right\|$, we have

$$
x_{1}<x_{1+u(n)}<x_{1+u(n-1)} \text {. }
$$

Now $x_{i}$ and $x_{i+u(n-1)}$ have the same sign for $0<i<u(n-2)$ by $\mathbf{H}_{n}$. Hence it follows by induction on $i$ that $x_{i+u(n)}$ lies between them and hence also has the same sign, for $i$ in this range. Since both $x_{i}$ and $x_{i+u(n-1)}$ have absolute value greater than $\left\|x_{u(n-1)}\right\|$ by $\mathbf{H}_{n}$, it follows also that $\left\|x_{i+u(n)}\right\|>$ $\left\|x_{u(n-1)}\right\|>\left\|x_{u(n)}\right\|$, for $i$ in this range. For $i=u(n-2)$, this argument proves that $x_{u(n-2)+u(n)}$ lies between $x_{u(n-2)}$ and $x_{u(n)}$ but does not determine its sign. However, it does follows that

$$
0<\left\|x_{u(n-2)+u(n)}\right\|<\left\|x_{u(n-2)}\right\| \text {, hence } x_{1}<x_{1+u(n-2)+u(n)}<x_{1+u(n-2)} .
$$

Now a similar inductive argument shows that $x_{i+u(n-2)+u(n)}$ lies between $x_{i}$ and $x_{i+u(n-2)}$ and hence has the required sign, for $0<i<u(n-3)$. Furthermore, this shows that $\left\|x_{i+u(n-2)+u(n)}\right\|>\left\|x_{u(n-1)}\right\|>\left\|x_{u(n)}\right\|$ for $i$ in this range. In the 
limiting case $i=u(n-3)$, this argument proves that $x_{i+u(n-2)+u(n)}=x_{u(n+1)}$ lies between $x_{u(n-3)}$ and $x_{i+u(n-2)}=x_{u(n-1)}$ but does not determine its sign. However, since $\left\|x_{u(n+1)}\right\|<\left\|x_{u(n-1)}\right\|<\left\|x_{u(n-3)}\right\|$, this proves that $x_{u(n-3)}$ and $x_{u(n-1)}$ have opposite sign, so that $x_{u(n-1)}$ also has the required sign. Thus, we have almost proved $\mathbf{H}_{n+1}$. The only missing pieces of information are the sign and magnitude of $x_{i}$ for $i=u(n-2)+u(n)$. then

We must prove that $\left\|x_{u(n-2)+u(n)}\right\|>\left\|x_{u(n)}\right\|$. But if $\left\|x_{u(n-2)+u(n)}\right\|<\left\|x_{u(n)}\right\|$

$$
x_{1}<x_{1+u(n-2)+u(n)}<x_{1+u(n)} .
$$

This is impossible, for a similar inductive argument would show that $x_{i+u(n-2)+u(n)}$ must be between $x_{i}$ and $x_{i+u(n)}$ for $0<i \leq u(n-2)$. In particular, taking $i=u(n-3)$ it would follow that $x_{u(n+1)}$ must be between $x_{u(n-3)}$ and $x_{u(n-3)+u(n)}$. By the part of $\mathbf{H}_{n+1}$ which has already been proved, these two have the same sign, and it would follow that $\left\|x_{u(n+1)}\right\|>\left\|x_{u(n)}\right\|$, which contradicts our hypothesis. Thus $\left\|x_{u(n-2)+u(n)}\right\|>\left\|x_{u(n)}\right\|$.

Now recall that $x_{u(n-2)+u(n)}$ is known to lie between $x_{u(n-2)}$ and $x_{u(n)}$. Since $\left\|x_{u(n-2)+u(n)}\right\|>\left\|x_{u(n)}\right\|$, it follows easily that $x_{u(n-2)+u(n)}$ has the same sign as $x_{u(n-2)}$. This completes the proof that $\mathbf{H}_{n} \Rightarrow \mathbf{H}_{n+1}$.

To show that this result is not vacuous, we must prove the following.

\section{Lemma 2.2. Fibonacci maps exist.}

We will outline two different proofs. (A third proof could be based on $\S 6$.) The proof below is an immediate application of the formal machinery of kneading theory, as developed in [MT]. An alternative proof, which is more direct and gives a more explicit description of the critical orbit, will be given in Lemma 3.1. Both proofs will make use of the following.

Definition 2.2. By a Fibonacci sum we will mean a finite or infinite formal sum

$$
\mu=u\left(n_{1}\right)+u\left(n_{2}\right)+u\left(n_{3}\right)+\cdots
$$

of nonconsecutive Fibonacci numbers. That is, we always assume that $n_{i+1} \geq$ $n_{i}+2$, with $n_{1} \geq 1$. It is not difficult to check that every positive integer has a unique expression as a finite Fibonacci sum. As an example, the difference $u(n)-1$ can be expressed as

$$
u(n)-1= \begin{cases}u(1)+u(3)+u(5)+\cdots+u(n-1) & \text { for } n \text { even } \\ u(2)+u(4)+u(6)+\cdots+u(n-1) & \text { for } n \text { odd }\end{cases}
$$

(For infinite Fibonacci sums, compare the proof of Lemma 3.1.)

As in [MT], we describe the kneading invariant of a unimodal map $f$ by a formal power series $D(t)=1+\varepsilon_{1} t+\varepsilon_{2} t^{2}+\cdots$, where each coefficient $\varepsilon_{n}$ is equal to +1 or -1 according as the function $x \mapsto\left|f^{\circ n}(x)\right|$ has a local minimum or local maximum at the origin. Since the $x_{i}$ are nonzero for $i>0$, we can check inductively that

$$
\varepsilon_{n}=\operatorname{sgn}\left(x_{1} x_{2} \cdots x_{n}\right)
$$


Such a kneading invariant is admissible (i.e., actually occurs) if and only if the inequality

$$
\sum_{0}^{\infty} \varepsilon_{i} t^{i} \leq \sum_{0}^{\infty}\left(\varepsilon_{m} \varepsilon_{m+i}\right) t^{i}
$$

is satisfied for every $m \geq 1$. Here, by definition, an inequality $\sum a_{i} t^{i}<\sum b_{i} t^{i}$ between formal power series means that the first difference $b_{i}-a_{i}$ which is nonzero is actually positive. Thus, for each $m$ we require that the smallest $i$ for which $\varepsilon_{m+i} \neq \varepsilon_{m} \varepsilon_{i}$ (if any such exist) must satisfy $\varepsilon_{i}=-1$.

In the case of a Fibonacci map, it follows inductively from (2.2), (2.3), and (2.5) that we must have $\varepsilon_{u(n)}=-1$ for every Fibonacci number $u(n)$. In fact, according to $(2.5), \varepsilon_{u(n+1)}$ is equal to $\varepsilon_{u(n)}$ multiplied by the sign of the product $x_{u(n)+1} x_{u(n)+2} \cdots x_{u(n+1)}$. This coincides with $\operatorname{sgn}\left(x_{1} x_{2} \cdots x_{u(n-1)}\right)=$ $\varepsilon_{u(n-1)}=-1$ except that the very last factor $x_{u(n-1)}$ has the wrong sign. Thus it follows inductively that $\varepsilon_{u(n)}=\varepsilon_{u(n+1)}=-1$ for all $n$. In other words, each map $x \mapsto\left|f^{\circ u(n)}(x)\right|$ must have a local maximum at $x=0$. For a $k$-fold Fibonacci sum

$$
m=u\left(n_{1}\right)+\cdots+u\left(n_{k}\right), \quad \text { where always } n_{1} \geq 1 \text { and } n_{i+1} \geq n_{i}+2
$$

Equations (2.2) and (2.5) imply that $\varepsilon_{m}$ equals the product $\varepsilon_{u\left(n_{1}\right)+\cdots+u\left(n_{k-1}\right)} \varepsilon_{u\left(n_{k}\right)}$. Hence it follows inductively that $\varepsilon_{m}=(-1)^{k}$. Thus, in order to prove Lemma 2.2 we need only show that the formal power series $\sum \varepsilon_{m} t^{m}$, with $\varepsilon_{m}$ defined by this equation, satisfies condition (2.6). That is, for each fixed $m$ the smallest $i$ with $\varepsilon_{m+i} \neq \varepsilon_{m} \varepsilon_{i}$ must satisfy $\varepsilon_{i}=-1$. However, if we express $m$ as a Fibonacci sum as above, then it is not hard to show that the smallest $i$ with $\varepsilon_{m+i} \neq \varepsilon_{m} \varepsilon_{i}$ is either $i=u\left(n_{1}-1\right)$ or $i=u\left(n_{1}\right)$ or (in the special case $n_{1}=1$ ) $i=2$. Since $\varepsilon_{i}=-1$ in each of these cases, the required inequality (2.6) follows. This completes the proof of Lemma 2.2.

Proof of Theorem 1.1. Since any unimodal kneading invariant which is admissible can be realized by a quadratic map, we can certainly find at least one quadratic map $f_{c}$ which realizes the given kneading invariant. (See, e.g., [MT].) But for any real quadratic map $f_{c}$ which is not infinitely renormalizable and has no attracting periodic orbit, Yoccoz has recently shown that the constant $c$ is uniquely determined by its kneading invariant. (This is an immediate corollary of his much more general result about complex quadratic parameter space.) Since it is easy to check that a quadratic Fibonacci map is not renormalizable and has no attracting periodic orbit, this proves Theorem 1.1.

\section{THE CRITICAL ORBIT}

Out of the kneading data, it is not difficult to determine the precise ordering of the points $x_{m}$ in the critical orbit. We can describe the resulting ordering by a fairly concrete model as follows. The construction will provide an alternative proof of Lemma 2.2 . 
Choose a parameter $0<t<1-t^{2}$, or in other words

$$
0<t<(\sqrt{5}-1) / 2=0.61803 \ldots \text {, }
$$

for example, $t=\frac{1}{2}$. Now for each integer $m \geq 0$, expressed as a Fibonacci sum (2.7), define a real number $y_{m}$ by the formula

$$
y_{m}= \pm\left(t^{n_{1}}-t^{n_{2}}+-\cdots \pm t^{n_{k}}\right)
$$

where the initial sign is to be -1 for $n_{1} \equiv 0,1(\bmod 4)$ and +1 for $n_{1} \equiv 2$, $3(\bmod 4)$, as in $(2.3)$. Thus the initial term $\pm t^{n_{1}}$ is the dominant one, and subsequent terms alternate in sign, decreasing by a factor of $t^{2}$ or more at each step since $n_{i+1} \geq n_{i}+2$.

Remark 3.1. More precisely, this ordering can be described as follows. For Fibonacci sums $m$ with different dominant terms, the order of the $y_{m}$ is determined by the rules

$$
y_{1+\ldots}<y_{5+\cdots}<y_{8+\cdots}<y_{34+\cdots}<\cdots<0<\cdots<y_{21+\ldots}<y_{13+\cdots}<y_{3+\cdots}<y_{2+\ldots} \text {. }
$$

Here, in each case, the dots in the subscript stand for higher terms, which may be zero, for an arbitrary Fibonacci sum. For two Fibonacci sums which have the same leading summands $u\left(n_{1}\right)+\cdots+u\left(n_{k}\right)$ but differ at the $(k+1)$ st summand, the relative order is determined as follows. Setting $s=u\left(n_{1}\right)+\cdots+u\left(n_{k}\right)$, we have

$$
\left|y_{s}\right|>\cdots>\left|y_{s+u\left(n_{k}+5\right)+\cdots}\right|>\left|y_{s+u\left(n_{k}+4\right)+\cdots}\right|>\left|y_{s+u\left(n_{k}+3\right)+\cdots}\right|>\left|y_{s+u\left(n_{k}+2\right)+\cdots}\right|
$$

if $k$ is odd and the same but with all inequalities reversed if $k \geq 2$ is even. Here all of these points $y_{s+\ldots}$ have the same sign, depending only on the leading summand $n_{1}$, as described above.

We claim that the resulting ordering of the $y_{m}$ is precisely the required ordering of the points $x_{m}$ in the critical orbit. More precisely, we will prove the following.

Lemma 3.1. The correspondence $y_{m} \mapsto y_{m+1}$ is unimodal, that is, it is monotone increasing on the set of $y_{m}$ for which $y_{m} \geq 0$ but monotone decreasing for $y_{m} \leq 0$. Furthermore, this correspondence is uniformly continuous. Thus, if we extend linearly over each gap between the $y_{m}$, then we obtain a continuous unimodal map $F$ from the interval $\left[y_{1}, y_{2}\right]$ to itself, satisfying the Fibonacci condition that

$$
y_{1}<y_{2}^{\prime}<y_{3}^{\prime}<y_{5}<y_{8}<y_{13}^{\prime}<\cdots<0 \text {, }
$$

where $y_{m}=F^{m}(0)$. (Here, as in $\S 2$, we use the notation $y \mapsto y^{\prime}$ for the orientation-reversing involution of the subinterval $\left[y_{2}^{\prime}, y_{2}\right]$ which satisfies the condition that $F\left(y^{\prime}\right)=F(y)$.)

Proof. It is convenient to divide the various $y_{m}$ into intervals $A_{n}, n \geq 0$, which are ordered according to the following pattern:

$$
A_{2}<A_{6}<A_{10}<\cdots<A_{8}<A_{4}<A_{0} \leq A_{1}<A_{5}<A_{9}<\cdots<A_{11}<A_{7}<A_{3} .
$$

(Here the two sequences $\left\{A_{2 n}\right\}$ and $\left\{A_{2 n+1}\right\}$ converge toward the two preimages of zero. Compare Corollary 3.4.) Let $A_{0}=\left[y_{5}, 0\right]$ be the closed interval 
containing all $y_{u(n)+\cdots}$ with $n \equiv 0,1(\bmod 4), n \geq 4$, and also containing the limit point zero. Here, as above, the notation $u(n)+\cdots$ stands for an arbitrary Fibonacci sum with leading term $u(n)$. Similarly, let $A_{1}=\left[0, y_{3}\right]$ be the interval containing all $y_{u(n)+\ldots}$ with $n \equiv 2,3(\bmod 4), n \geq 3$, together with the limit point zero. For $n \geq 2$ even, let $A_{n}$ be the smallest interval containing all $y_{m}$ with $m$ of the form $u(1)+u(3)+\cdots+u(n-1)+$ (higher terms), where the higher terms if any must start with $u(n+2)$ or higher. Using the identity (2.4), it follows easily that $A_{n}$ is equal to the closed interval spanned by the two points $y_{u(n)-1}$ and $y_{u(n)+u(n+2)-1}$. Here the relative order of these two end points depends on whether $n$ is congruent to 0 or 2 modulo 4. Similarly, for $n \geq 3$ odd, we define $A_{n}$ to be the smallest interval containing all $y_{m}$ with $m$ of the form $u(2)+u(4)+\cdots+u(n-1)+$ (higher), where again the higher summands if any must start with $u(n+2)$ or higher. Again using the identity (2.4), we see that this interval $A_{n}$ is again spanned by the points $y_{u(n)-1}$ and $y_{u(n)+u(n+2)-1}$, where the relative order of the two end points depends on whether $n$ is congruent to 1 or 3 modulo 4 .

It is not difficult to show that every $y_{m}$ with $m>0$ belongs to exactly one of these intervals and that these points are ordered according to the pattern described above. For $y_{m} \in A_{n}$ a brief computation shows that the map $y_{m} \mapsto$ $y_{m+1}$ is linear with slope $(-1)^{n-1}$. In particular, it is either order preserving or order reversing according as $A_{n} \subset\left[0, y_{2}\right]$ or $A_{n} \subset\left[y_{1}, 0\right]$. If we extend this map to be linear in the gap between $A_{n}$ and $A_{n+4}$, then computation shows that the slope in this gap takes the value

$$
\frac{\Delta F(x)}{\Delta x}=(-1)^{n-1} \frac{t^{n}-t^{n+2}-t^{n+4}}{t^{n+1}-t^{n+2}-t^{n+3}}
$$

for $n>0$. This is independent of $n$ except for sign. For $n=0$ it takes a different value but still with the appropriate (negative) sign. As an example, for $t=\frac{1}{2}$ this gap slope is equal to $\pm \frac{11}{2}$ for $n>0$ and is $-\frac{6}{5}$ for $n=0$. In this way, we obtain the required explicit unimodal map $F$ which realizes the given kneading data. This proves Lemma 3.1, and completes the alternate proof of Lemma 2.2 .

Lemma 3.2. If the Fibonacci map $f$ has no "homtervals" within the interval $\left[x_{1}, x_{2}\right]$, that is, if the precritical points are everywhere dense, then $f$ restricted to this interval is topologically conjugate to this model map $F$.

The proof is straightforward.

Remark 3.2. By definition, a homterval is a subinterval of $I$ which is mapped homeomorphically by all iterates of $f$. A wandering interval is a homterval which is not contained in the basin of attraction for any periodic orbit. According to Guckenheimer [G1], a unimodal map has no wandering intervals within $\left[x_{1}, x_{2}\right]$ provided that it has negative Schwarzian, with nonflat critical point. According to de Melo and van Strien [MvS], it has no wandering intervals provided that it is sufficiently smooth, with nonflat critical point. (See also Blokh and Lyubich [L1, BL1].) 
Lemma 3.3. More generally, if a Fibonacci map has no wandering intervals, then its critical orbit closure $\overline{\mathscr{O}}$ is a Cantor set, homeomorphic to the corresponding critical orbit closure for the model map $F$. In particular, this Cantor set is canonically homeomorphic to the set of all finite or infinite Fibonacci sums, suitably topologized.

Proof of 3.3. The appropriate topology for the set of all finite or infinite Fibonacci sums can be described as follows. Let $\Sigma$ be the "Fibonacci shift", consisting of all sequences $\left(a_{1}, a_{2}, \ldots\right)$ of zeros and ones with no two consecutive ones. In other words, $\Sigma$ is a one-sided subshift of finite type corresponding to the matrix $T=\left(\begin{array}{ll}1 & 1 \\ 1 & 0\end{array}\right)$. (The name is suggested since the number of cylinders in $\Sigma$ of length $n$ is equal to $u(n+1)$.) This set $\Sigma$ is topologized as a subset of the infinite Cartesian product $\{0,1\} \times\{0,1\} \times \cdots$. Each sequence $\left\{a_{n}\right\} \in \Sigma$ determines an associated Fibonacci sum $\mu=\sum a_{n} u(n)$, and we give the set consisting of all Fibonacci sums the corresponding compact topology. It is easy to check that the correspondence $m \mapsto x_{m}$, where $m$ ranges over positive integers expressed as finite Fibonacci sums, extends uniquely to a homeomorphism $\mu \mapsto x_{\mu} \in \overline{\mathscr{O}}$, where now $\mu$ ranges over finite or infinite Fibonacci sums. Further details of the proof are straightforward.

Remark 3.3. It is sometimes convenient to order partially the Cantor set $\Sigma$ using lexicographical order from the right. Thus two sequences of zeros and ones, with no two consecutive ones, are comparable whenever they are eventually equal or, in other words, have the same tail. In terms of this ordering, the map from $\Sigma$ to itself which corresponds to the map $f \mid \overline{\mathscr{O}}$ can be described as the immediate successor function, which carries each such sequence to the next largest sequence with the same tail (such a transformation is called an adic shift, compare [VL]). However, there are two exceptional sequences which are maximal and hence have no successor, immediate or otherwise, namely, the two sequences $(1,0,1,0, \ldots)$ and $(0,1,0,1, \ldots)$ corresponding to the Fibonacci sums $1+3+8+\cdots$ and $2+5+13+\cdots$ respectively. These both map to the zero sequence. (Compare (2.4).)

Corollary 3.4. The mapping $f$ from the Cantor set $\overline{\mathscr{O}}$ onto itself is one-to-one except that the point zero has two different pre-images, corresponding to the infinite Fibonacci sums $u(1)+u(3)+u(5)+\cdots$ and $u(2)+u(4)+u(6)+\cdots$.

The proof is straightforward.

Here is a more explicit description of this Cantor set as a subset of the real line. For each $n \geq 1$ let $I^{n} \subset \mathbf{R}$ be the smallest closed interval containing all of the points $x_{u(q)}$ with $q \geq n$. Thus $I^{n}$ is a closed neighborhood of the origin. One end point of this interval is $x_{u(n)}$ and the other end point is either $x_{u(n+1)}$ or $x_{u(n+2)}$ according as $n$ is odd or even. Note that the map $f$ folds $I^{n}$ over onto the closed interval $\left[x_{1}, x_{u(n)+1}\right]$, which in turn maps onto the closed interval $\left[x_{u(n)+2}, x_{2}\right]$ provided that $n \geq 3$. For each $k \geq 0$, we will use the notation $I_{k}^{n}$ for the image $f^{k}\left(I^{n}\right)$. According to $\S 2$, this image $I_{k}^{n}$ is disjoint from the origin for $1 \leq k<u(n-1)$ but contains the origin for $k=u(n-1)$. However, $I_{u(n-1)}^{n}$ contains a smaller interval $I_{u(n-1)}^{n+1}$ which again is disjoint 
from the origin. It will be convenient to use the notation

$$
J^{n}=I_{u(n-1)}^{n+1}, \quad \text { and more generally, } \quad J_{k}^{n}=f^{k}\left(J^{n}\right)=I_{k+u(n-1)}^{n+1} .
$$

Note, in particular, that $J_{u(n-2)}^{n}=I_{u(n)}^{n+1}$.

Definition 3.1. Let $M^{n}$ be the $u(n)$-fold union

$$
M^{n}=\bigcup_{0 \leq k<u(n-1)} I_{k}^{n} \cup \bigcup_{0 \leq k<u(n-2)} J_{k}^{n} .
$$

For example (lising the subintervals from left to right),

$$
\begin{aligned}
& M^{1}=\left[x_{1}, x_{2}\right], \\
& M^{2}=\left[x_{1}, x_{4}\right] \cup\left[x_{5}, x_{2}\right], \\
& M^{3}=\left[x_{1}, x_{4}\right] \cup\left[x_{5}, x_{3}\right] \cup\left[x_{7}, x_{2}\right], \\
& M^{4}=\left[x_{1}, x_{6}\right] \cup\left[x_{12}, x_{4}\right] \cup\left[x_{5}, x_{13}\right] \cup\left[x_{11}, x_{3}\right] \cup\left[x_{7}, x_{2}\right],
\end{aligned}
$$

and so on.

Lemma 3.5. The $u(n)$ closed intervals

$$
I_{0}^{n}, I_{1}^{n}, \ldots, I_{u(n-1)-1}^{n} \text { and } J_{0}^{n}, \ldots, J_{u(n-2)-1}^{n}
$$

are pairwise disjoint. Denoting their union by $M^{n}$ as above, the $M^{n}$ form a nested sequence of closed sets $M^{1} \supset M^{2} \supset M^{3} \supset \cdots$ with intersection equal to the Cantor set $\mathbb{O}$.

Proof. We will show by induction on $n$ that the $u(n)$ subintervals of $M^{n}$ are pairwise disjoint, that the $M^{n}$ are nested, and that each $M^{n}$ contains the critical orbit closure. The idea of the proof is to show that, as we pass from $M^{n}$ to $M^{n+1}$, each of the $u(n-1)$ intervals $I_{k}^{n} \subset M^{n}$ will be replaced by two subintervals $I_{k}^{n+1}$ and $J_{k}^{n+1}$ in $M^{n+1}$, while each of the $u(n-2)$ intervals $J_{k}^{n}=I_{k+u(n-1)}^{n+1}$ remains unchanged.

To start the induction, it is trivially true that $M^{1}=\left[x_{1}, x_{2}\right]$ contains the critical orbit closure. The first step in the induction is to note that each $I^{n}$ contains $I^{n+1}$ and $J^{n+1}$ as disjoint subsets. For example, if $n \equiv 3(\bmod 4)$ then these two subintervals of

$$
I^{n}=\left[x_{u(n+1)}, x_{u(n)}\right]
$$

are situated as shown in Figure 1.

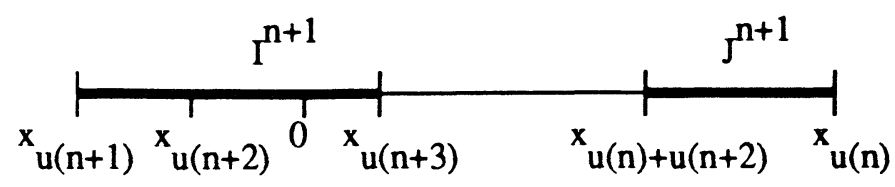

FIGURE 1. The interval $I^{n}$ in the case $n \equiv 3(\bmod 4)$. 
The picture for $n \equiv 1(\bmod 4)$ is a mirror image, and the pictures for $n \equiv$ $0,2(\bmod 4)$ are quite similar. Note that the map $f^{u(n)}$ folds the subinterval $I^{n+2} \subset I^{n+1}$ over onto $J^{n+1}$, while the map $f^{u(n-1)}$ carries $J^{n+1}$ back onto a neighborhood of the origin, spanned by the two points $x_{u(n+1)}$ and $x_{u(n+3)}$. In the case $n \equiv 3(\bmod 4)$ as illustrated, $I^{n+2}$ is the interval $\left[x_{u(n+2)}, x_{u(n+3)}\right]$, while the image $f^{\circ u(n-1)}\left(J^{n+1}\right)=\left[x_{u(n+1)}, x_{u(n+3)}\right]$ coincides with the interval $I^{n+1}$

It follows easily from Remark 3.1 and Lemma 3.3 that the two subintervals

$$
I^{n+1}, J^{n+1} \subset I^{n}
$$

are indeed disjoint and together contain all of the points of $\overline{\mathscr{O}} \cap I^{n}$. For $1 \leq$ $k<u(n-1)$, a similar argument shows that the two subintervals

$$
I_{k}^{n+1}, J_{k}^{n+1} \subset I_{k}^{n}
$$

are disjoint and together contain all of the points of $\overline{\mathscr{O}} \cap I_{k}^{n}$. This completes the induction and shows that

$$
M^{1} \supset M^{2} \supset M^{3} \supset \cdots \supset \overline{\mathscr{\sigma}} \text {. }
$$

Since each end point of each subinterval of $M^{n}$ belongs to the orbit $\mathscr{O}$, using the hypothesis that there are no wandering intervals we see easily that $\cap M^{n}$ is equal to $\overline{\mathscr{O}}$.

Using the sets $M^{n}$ one can give another description of the above correspondence between $\overline{\mathscr{O}}$ and $\Sigma$ (see Lemma 3.3). Given $x \in \overline{\mathscr{O}}$, let $M^{n}(x)$ be an interval of the set $M^{n}$ containing $x$. Then set $a_{n}=0$ if $M^{n}(x)=I_{k}^{n}$ and $a_{n}=1$ if $M^{n}(x)=I_{k}^{n+1}$ (for appropriate $k$ 's). One can check that $\left\{a_{n}\right\} \in \Sigma$ is the sequence corresponding to $x \in \overline{\mathscr{O}}$.

In what follows we will use the notation $M_{a_{1} \cdots a_{n}}^{n}$ for the interval of $M^{n}$ corresponding to the cylinder $\left[a_{1} \cdots a_{n}\right] \subset \Sigma$.

Lemma 3.6. Still assuming that there are no wandering intervals, the points $x_{i}$, $i \geq 1$, are the end points of the complementary intervals for the critical orbit closure $\overline{\mathscr{O}} \subset \mathbf{R}$. More explicitly, the Cantor set $\mathscr{\mathscr { O }}$ can be obtained from the closed interval $\left[x_{1}, x_{2}\right]$ by removing a dense collection of disjoint open subintervals $\left(x_{p}, x_{q}\right)$ as follows. If one of $p, q$ is a Fibonacci sum of the form

$$
u\left(n_{1}\right)+\cdots+u\left(n_{k-1}\right)+u\left(n_{k}\right)+u\left(n_{k}+2\right)
$$

with $k \geq 2$, then the other is equal to

$$
u\left(n_{1}\right)+\cdots+u\left(n_{k-1}\right)+u\left(n_{k}+1\right)
$$

On the other hand, if one is $u(n)+u(n+2)$, then the other is either $u(n+1)$ or $u(n+3)$ according as $n$ is even or odd.

As an example, the first seven open subintervals to be removed are as follows, in their natural order:

$$
\left(x_{9}, x_{19}\right) \cup\left(x_{6}, x_{12}\right) \cup\left(x_{4}, x_{5}\right) \cup\left(x_{18}, x_{8}\right) \cup\left(x_{13}, x_{11}\right) \cup\left(x_{3}, x_{7}\right) \cup\left(x_{20}, x_{10}\right) \text {. }
$$


In other words, the Cantor set $\overline{\mathscr{O}}$ is contained in the disjoint union

$$
\begin{aligned}
{\left[x_{1}, x_{9}\right] \cup\left[x_{19}, x_{6}\right] \cup\left[x_{12}, x_{4}\right] \cup\left[x_{5}, x_{18}\right] } \\
\cup\left[x_{8}, x_{13}\right] \cup\left[x_{11}, x_{3}\right] \cup\left[x_{7}, x_{20}\right] \cup\left[x_{10}, x_{2}\right]
\end{aligned}
$$

(which coincides with the closed set $M^{5}$ ). The proof of this statement is a straightforward consequence of the ordering of the points in the critical orbit, as described above.

We can obtain a different model for this critical orbit closure as follows. Let

$$
\gamma=(1-\sqrt{5}) / 2=-.61803 \ldots,
$$

so that $\gamma=\gamma^{2}-1$. To each finite or infinite Fibonacci sum $\mu=u\left(n_{1}\right)+u\left(n_{2}\right)+$ $\cdots$, let us assign the real number $\phi\left(x_{\mu}\right)=\gamma\left(\gamma^{n_{1}}+\gamma^{n_{2}}+\cdots\right)$ modulo one.

Lemma 3.7. The resulting map $\phi$ from the critical orbit closure $\overline{\mathscr{O}}$ onto the circle $\mathbf{R} / \mathbf{Z}$ is one-to-one except at the countably many iterated pre-images of zero. It semiconjugates the map $f \mid \overline{\mathscr{O}}$ onto the golden rotation $t \mapsto t+\gamma(\bmod 1)$.

Proof. It is easy to check that $\phi$ is well defined and continuous. Note that the identity $u(n-1)+u(n)=u(n+1)$ corresponds to the identity $\gamma^{n-1}+\gamma^{n}=\gamma^{n+1}$. Using this fact, it is not difficult to check the required identity

$$
\phi\left(f\left(x_{\mu}\right)\right)=\phi\left(x_{\mu+1}\right) \equiv \phi\left(x_{\mu}\right)+\gamma \quad(\bmod 1) .
$$

Thus the image is a compact subset of the circle, invariant under the golden rotation, and hence is equal to the entire circle. Now consider any Fibonacci sum with leading term $u(n)$. A brief computation shows that the corresponding image

$$
\phi\left(x_{u(n)+\cdots}\right)=\gamma^{n+1}+\cdots
$$

lies somewhere between

$$
\gamma^{n+1}+\gamma^{n+3}+\gamma^{n+5}+\cdots=\gamma^{n+1} /\left(1-\gamma^{2}\right)=-\gamma^{n}
$$

and

$$
\gamma^{n+1}+\gamma^{n+4}+\gamma^{n+6}+\cdots=\gamma^{n+1}-\gamma^{n+3}=-\gamma^{n+2}
$$

Thus, depending on the leading summand, the image $\phi\left(x_{\mu}\right)$ lies in one of the nonoverlapping intervals

$$
\begin{gathered}
{\left[-\gamma^{2},-\gamma^{4}\right] \cup\left[-\gamma^{4},-\gamma^{6}\right] \cup\left[-\gamma^{6},-\gamma^{8}\right] \cup \cdots \cup\{0\} \cup \cdots} \\
\cup\left[-\gamma^{7},-\gamma^{5}\right] \cup\left[-\gamma^{5},-\gamma^{3}\right] \cup\left[-\gamma^{3},-\gamma\right],
\end{gathered}
$$

having total length $-\gamma-\left(-\gamma^{2}\right)=1$. Hence the value $\phi\left(x_{\mu}\right) \in \mathbf{R} / \mathbf{Z}$ determines the leading summand $u(n)$ uniquely, except in countably many cases which can be explicitly described. For two Fibonacci sums with the same leading term, a similar argument shows that the value $\phi\left(x_{\mu}\right)$ determines the second term uniquely, again with the exception of countably many cases which can be explicitly described; and a similar argument applies to higher terms. 
Corollary 3.8. With hypotheses as above, the map $f \mid \overline{\mathscr{O}}$ is minimal, that is, every orbit is dense, and has topological entropy zero. Furthermore this map is uniquely ergodic, that is, it has one and only one invariant probability measure.

Proof. This follows easily from the corresponding assertion for an irrational rotation of the circle.

Combining the results of this section, this evidently completes the proof of Theorem 1.2.

\section{A PRIORI BOUNDS}

In the following two sections we assume that $f:[-1,1] \rightarrow[-1,1]$ is a $C^{2}$ smooth unimodal map with nondegenerate minimum point 0 , and normalized by the condition $f(-1)=f(1)=1$ (which does not restrict the generality). Denote the class of maps by $\mathscr{U}$, and let us discuss topology on this space.

We will mainly be interested in the subspace $\mathscr{U}_{0} \subset \mathscr{U}$ consisting of those $f$ for which $f$ is an even function, $f(-x)=f(x)$. We will first discuss the differentiability conditions and topology on this subspace and then generalize to the full space $\mathscr{U}$. If $f$ is even, then we can write it uniquely as

$$
f(x)=A_{x_{1}} \circ g \circ Q
$$

where $Q$ is the squaring map $\xi \mapsto \xi^{2}, g$ is some orientation-preserving diffeomorphism of $[0,1]$, and $A_{x_{1}}$ is the orientation-preserving affine map which carries $[0,1]$ onto $\left[x_{1}, 1\right]$, where $x_{1}=f(0)$ is the critical value.

Now the $C^{k}$-topology on $\mathscr{U}_{0}, k \leq 2$, comes from the $C^{k}$-topology on the space of diffeomorphisms $g$, together with the line topology on the range of the parameter $x_{1}$. Let $\|f\|$ denote the maximum of the $C^{2}$-norms for $g, g^{-1}$ which is a continuous functional in $C^{2}$-topology on our space.

To obtain a corresponding topology of the full space $\mathscr{U}$, we need one extra step. Let $x \mapsto x^{\prime}$ be the orientation-reversing diffeomorphism of $T$ which satisfies $f(x)=f\left(x^{\prime}\right)$. This involution is certainly $C^{2}$-smooth. Consider a map $B: x \mapsto\left(x-x^{\prime}\right) / 2$. Evidently $f$ can be expressed as a function of $\left(x-x^{\prime}\right)^{2} / 4$, so that we have a presentation $f(x)=A_{x_{1}} \circ g \circ Q \circ B$ instead of that above. Now we must incorporate the $C^{k}$ topology on the involution as part of our topology. In practice, it is easiest simply to carry out this symmetrizing change of coordinate $x \mapsto\left(x-x^{\prime}\right) / 2$ in the beginning and thereafter to deal only with even maps $f$. Moreover, we can also assume without loss of generality that $f$ is purely quadratic $x \mapsto x^{2}-c$ near 0 (since any $f \in \mathscr{U}$ is $C^{2}$-conjugate to such one).

Denote by $\mathscr{F}$ the subspace of Fibonacci maps $f \in \mathscr{U}$.

The following notation will be used throughout the paper:

$$
d_{n}=\left|x_{u}(n)\right|, \quad \lambda_{n}=d_{n} / d_{n-1} .
$$

The goal of this section is to obtain some a priori estimates for the $\lambda_{n}$ (compare $[\mathrm{G} 2, \mathrm{~L} 1, \mathrm{BL} 3, \mathrm{M}, \mathrm{S}]$, etc.). The proofs are based upon the Schwarz lemma and the Koebe principle, both of which are stated in the appendix. 
First let us introduce convenient terminology and notation. A family of intervals $\mathbf{G}=\left\{G_{i}\right\}_{i=0}^{n}$ is called a chain of intervals if $G_{i}$ is a component of $f^{-1} G_{i+1}$ for $i=0,1, \ldots, n-1$. The chain is called monotone if all maps $f: G_{i} \rightarrow G_{i+1}$ are homeomorphisms.

For a given interval $G$ and a point $x$ such that $f^{n} x \in G$ one can construct a chain $G_{0}, G_{1}, \ldots, G_{n} \equiv G$ pulling $G$ back along the $n$-orbit of $x$. This construction is an efficient tool in one-dimensional dynamics because it is often possible to estimate the distortion of $f^{n}$ along chains of intervals (see [L1, S]).

For a family of intervals $\mathbf{G}=\left\{G_{i}\right\}$ denote by $|\mathbf{G}|=\Sigma\left|G_{i}\right|$ the total length of intervals $G_{i}$ and by mult $\mathbf{G}$ the maximal intersection multiplicity of intervals $G_{i}$, that is, the maximum number of $G_{i}$ having nonvacuous intersection.

Let us consider now the pull-back

$$
\mathbf{H}^{n+1}=\left\{H_{m}^{n+1}\right\}_{m=0}^{u(n)-1}, \quad H_{0}^{n+1} \equiv H^{n+1} \supset I_{1}^{n+1}
$$

of the interval $T^{n-2}$ along the orbit $\left\{f^{m} I_{1}^{n+1}\right\}_{m=0}^{u(n)-1}$. The following two topological lemmas easily follow from the above combinatorics.

Lemma 4.1. The chain $\mathbf{H}^{n+1}$ is monotone (so that $f^{n}$ monotonically maps $H^{n+1}$ onto $T^{n-2}$ ).

Let us consider any interval $I=I_{k}^{l}, l \in\{n, n+1\}$, of the family $M^{n}$ different from $I_{0}^{n}, I_{1}^{n}, I_{2}^{n}$. Define an interval $F \equiv F_{n}(I) \supset I$ as follows:

(i) If $I \neq J^{n}$ then $F$ is the convex hull of two neighbors of $I$ in the family $M^{n}$

(ii) If $I=J^{n}$ then $F$ is the half of the interval $T^{n-2}$ containing $I$. Now consider the pull-back $\mathbf{G}=\left\{G_{i}\right\}_{i=0}^{k}$ of $F \equiv G_{k}$ along the $k$-orbit of $I_{0}^{l}$.

Lemma 4.2. Under the above circumstances

(1) $\left\{G_{i}\right\}_{i=1}^{k}$ is a monotone chain of intervals;

(2) $G_{0} \subset T^{l-1}$.

Lemma 4.3. The intersection multiplicities of the above chains $\mathbf{G}$ and $\mathbf{H}^{n+1}$ are uniformly bounded:

$$
\text { mult } \mathbf{G} \leq 8 \text { and mult } \mathbf{H}^{n+1} \leq 8 .
$$

Proof. If $t$ intervals of the chain $\left\{G_{i}\right\}_{i=1}^{k}$ have a common point, then there is an interval $G_{i}$ among them containing at least $(t-1) / 2$ intervals $N_{s}$ of the $(k-1)$-orbit of $I_{1}^{l}$. Since $f^{k-i} \mid G_{i}$ is monotone, $f^{k-i} N_{s}$ belongs to the $(u(l-1)-1)$-orbit of $I_{1}^{l}$. But $G_{k}$ contains at most three intervals of this orbit. Hence $t \leq 7$.

The argument for $\mathbf{H}$ is similar, and we omit it.

Now we have enough topological information for getting a priori bounds.

Lemma 4.4. $\sup _{n} \lambda_{n} \lambda_{n+1}<1$.

Proof. Choose the smallest interval $I$ among $\left[0, x_{u(n)}\right]$ and $I_{k}^{l} \in M^{n}$ with $k>0$. It is easy to analyze the cases $I=\left[0, x_{u(n)}\right]$ or $I=I_{k}^{n}$ for $k=1,2$. 
So, we restrict ourselves to other cases, and then the interval $F$ is well defined. Moreover, the Poincaré length $[I: F]$ does not exceed $\log 4$.

It follows from Lemmas 4.2(1), 4.3 and the Schwarz lemma that the Poincare length $\left[I_{1}^{l}: G_{1}\right]$ is uniformly bounded (by a constant depending on $\|f\|$ ). Since $f$ is quadratic (and hence quasi-symmetric) near the critical point, the ratio

$$
\frac{\left|G_{0}\right|}{\left|G_{0} \backslash T^{l}\right|}
$$

can be estimated through $\left[I_{1}^{l}: G_{1}\right]$, and hence the ratio $\left|T^{l}\right| /\left|G_{0}\right|$ is bounded away from 1.

By Lemma 4.2(2), $G_{0} \subset T^{l-1}$, so $\lambda_{l} \leq\left|T^{l}\right| /\left|G_{0}\right|$. It remains to mention that $\lambda_{l}$ is equal to either $\lambda_{n}$ or $\lambda_{n+1}$.

Lemma 4.5.

$$
\frac{1}{1-\lambda_{n+1}^{2}} \leq\left(\frac{1+\lambda_{n} \lambda_{n-1}}{1-\lambda_{n} \lambda_{n-1}}\right)^{2}\left(1+O\left(\left|\mathbf{H}^{n+1}\right|\right)\right) .
$$

Proof. Applying the Schwarz lemma to the monotone map

$$
f^{u(n)-1}:\left(H^{n+1}, I_{1}^{n+1}\right) \rightarrow\left(T^{n-2}, I_{0}^{n}\right)
$$

we get

$$
\left[I_{1}^{n+1}: H^{n+1}\right] \leq\left[T^{n}: T^{n-2}\right]+O\left(\left|\mathbf{H}^{n+1}\right|\right)=2 \log \frac{1+\lambda_{n} \lambda_{n-1}}{1-\lambda_{n} \lambda_{n-1}}+O\left(\left|\mathbf{H}^{n+1}\right|\right)
$$

Let $G$ be the component of $f^{-1} H^{n+1}$ containing $0, \mu=\left|T^{n+1}\right| /|G|$. The calculation for the quadratic map shows that

$$
\log \frac{1}{1-\mu^{2}} \leq\left[I_{1}^{n+1}: H^{n+1}\right]
$$

Furthermore, since $f^{u(n)}$ is not unimodal on $T^{n}, G \subset T^{n}$. Hence $\lambda_{n+1} \leq \mu$. The last estimate together with (4.2) and (4.3) yield the required.

From Lemmas 4.4 and 4.5 we get immediately an a priori bound of $\lambda_{n}$ :

Lemma 4.6. $\sup _{n} \lambda_{n}<1$.

Lemma 4.7. Let $L^{n}$ be the gap between $T^{n}$ and $J^{n}$. Then

$$
\sup _{n} \frac{\left|L^{n}\right|}{\left|x_{u(n)}\right|}<1 .
$$

Proof. Because of Lemma 4.6, it is enough to show that the gap $L$ is not too small as compared with $J^{n}$. Let $N$ be a monotonicity interval of $f^{u(n-2)}$ adjacent to $J^{n}$ on its outer side. Consider the map $f^{u(n-2)} \mid L \cup J^{n} \cup N$ and apply to it the Schwarz lemma taking into account Lemmas 4.1 and 4.6.

Now we can prove that the Lebesgue measure of $M^{n}$ and $\mathbf{H}^{n}$ go down exponentially fast (compare $[\mathrm{G} 2, \mathrm{BL} 3, \mathrm{~S}]$ ). Let $[[\alpha, \beta]]$ denote the smallest closed interval containing both $\alpha$ and $\beta$ (similarly, $((\alpha, \beta))$ will denote the smallest open interval containing $\alpha$ and $\beta$ ). 
Lemma 4.8. There exist constants $C>0$ and $q<1$ such that

$$
\left|\mathbf{H}^{n}\right| \leq C q^{n} \text { and }\left|M^{n}\right| \leq C q^{n} \text {. }
$$

Hence, the Lebesgue measure of $\omega(0)$ is equal to zero.

Remark 4.1. The last statement is a corollary of more general results [BL2, M].

Proof. By Lemma 4.7, density of $M^{n+1}$ in $I_{0}^{n}$ is bounded away from 1. Consider now an interval $I_{l}^{n} \in M^{n}, l>0$. It follows from Lemmas 4.1 and 4.6 that the map

$$
f^{u(n-1)-l}: I_{l}^{n} \rightarrow\left[\left[x_{u(n-1)}, x_{u(n+1)}\right]\right]
$$

has bounded distortion. But this map carries $M^{n+1} \cap I_{l}^{n}$ into $I_{0}^{n+1} \cup J^{n}$. By Lemma 4.7, density of the latter set in $\left[\left[x_{u(n-1)}, x_{u(n+1)}\right]\right]$ is bounded away from 1 . Hence density of $M^{n+1}$ in $I_{l}^{n}$ is bounded away from 1 as well. So, there is a $q<1$ such that

$$
\lambda\left(M^{n+1}\right) \leq q \lambda\left(\bigcup_{l=0}^{u(n-1)-1} I_{l}^{n}\right)+\lambda\left(\bigcup_{l=u(n-1)}^{u(n)-1} I_{l}^{n+1}\right) .
$$

Applying this estimate twice we get

$$
\lambda\left(M^{n+2}\right) \leq q \lambda\left(M^{n}\right)
$$

and we are done with $M^{n}$.

Now consider a pair $H^{n+1} \supset H^{n+2}$ and apply $f^{u(n)-1}$. Then $H^{n+1}$ is mapped onto $T^{n-2}$, while $H^{n+2}$ is mapped into $T^{n-1}$ (since $f^{u(n-1)}$ is monotone on its image). By Lemma 4.6 and the Schwarz lemma, the density of $f^{m} H^{n+2}$ in $f^{m} H^{n+1}$ is bounded away from 1 for $m=0, \ldots, u(n)-1$. Furthermore,

$$
f^{u(n)+m} H^{n+2} \subset I_{m}^{n-1}, \quad m=1, \ldots, u(n-1) .
$$

Consequently, for some $q_{1}<1$ we have

$$
\left|\mathbf{H}^{n+2}\right| \leq q_{1}\left|\mathbf{H}^{n+1}\right|+\left|M^{n-1}\right|+\left|M^{n-2}\right|
$$

and the required follows.

Lemma 4.9. (i). There is a $q<1$ such that $\lambda_{n+1}^{2}=O\left(\lambda_{n} \lambda_{n-1}+q^{n}\right)$.

(ii). $\lambda_{n+1}^{2}=O\left(\left|J^{n}\right| /\left|T^{n-1}\right|\right)$.

Proof. The point (i) follows from Lemmas 4.5 and 4.8. To prove (ii), consider $f^{u(n-1)}: I^{n+1} \rightarrow J^{n}$ and apply the Schwarz lemma.

Remark 4.2. All constants in the above estimates depend only on $\|f\|$. Moreover, they are uniform over the maps with negative Schwarzian derivative (since the Schwarz lemma and the Koebe principle are uniform over this class). Finally, all estimates are asymptotically uniform over the whole class $\mathscr{U}$ ("beau estimates", see Sullivan [S]). For example, Lemma 4.6 can be improved in such a way:

$$
\limsup _{n \rightarrow \infty} \lambda_{n} \leq C<1
$$

for an absolute constant $C$. 


\section{Scaling, Characteristic EXPONENT, AND HAUSDORFF DIMENSION}

In this section we will prove Theorem 1.3 assuming that there is a good enough a priori bound of $\lambda_{n}$. It follows that Theorem 1.3 holds for an open set of Fibonacci maps invariant under quasi-symmetric conjugacy.

Let $q<1$ be the constant from Lemma 4.8, $\sigma_{n}=\max _{n-1 \leq i \leq n+1}\left(\lambda_{i}, \lambda_{i+1}\right)$.

Lemma 5.1. For any $x \in I_{1}^{n+1}$

$$
\frac{d_{n}}{d_{n+1}^{2}}\left(1+O\left(\sigma_{n}+q^{n}\right)\right)^{-1} \leq\left|\left(f^{u(n)-1}\right)^{\prime}(x)\right| \leq \frac{d_{n}}{d_{n+1}^{2}}\left(1+O\left(\sigma_{n}+q^{n}\right)\right) .
$$

Proof. Let us apply the Koebe Principle to the map

$$
f^{u(n)-1}:\left(H^{n+1}, I^{n+1}\right) \rightarrow\left(T^{n-2}, T^{n}\right)
$$

taking into account Lemma 4.8

$$
\frac{\left|\left(f^{u(n)-1}\right)^{\prime}(x)\right|}{\left|\left(f^{u(n)-1}\right)^{\prime}(y)\right|}=1+O\left(\lambda_{n} \lambda_{n-1}+q^{n}\right), \quad x, y \in I_{1}^{n+1} .
$$

Besides,

$$
\frac{d_{n}}{d_{n+1}^{2}} \leq \frac{\left|I^{n}\right|}{\left|I_{1}^{n+1}\right|} \leq\left(1+\lambda_{n+1} \lambda_{n+2}\right) \frac{d_{n}}{d_{n+1}^{2}}
$$

and the lemma follows.

Lemma 5.2. There is a $\rho=\rho(\|f\|)$ and $L=L(\|f\|) \in \mathbf{N}$ such that if $\lambda_{l}<\rho$ for some $l \geq L$ then $\lambda_{n}$ exponentially decrease. For maps with nonpositive Schwarzian derivative one can choose $L=1$ and uniform $\rho$.

Proof. Let $n$ be so large that $f(x)$ is a quadratic map in the neighborhood $T_{n-1}$. Then by the chain rule,

$$
\left|\left(f^{u(n)-1}\right)^{\prime}\left(x_{1}\right)\right|=\left|\left(f^{u(n-1)-1}\right)^{\prime}\left(x_{1}\right)\right| \cdot 2 d_{n-1}\left|\left(f^{u(n-2)-1}\right)^{\prime}\left(x_{u(n-1)+1}\right)\right| .
$$

By Lemma 5.1,

$$
\frac{d_{n-1}}{d_{n}^{2}} \cdot 2 d_{n-1} \frac{d_{n-2}}{d_{n-1}^{2}} \leq \frac{d_{n}}{d_{n+1}^{2}}\left(1+O\left(\sigma_{n-2}+\sigma_{n-1}+\sigma_{n}+q^{n}\right)\right) .
$$

It follows from Lemma 4.5 that $\lambda_{k}$ remains small for $k=n-2, \ldots, n+1$, once $\lambda_{n-3}$ becomes small for big enough $n$. Hence, by (5.2)

$$
\lambda_{n+1}^{2} \leq \gamma \lambda_{n} \lambda_{n-1}
$$

for some $\gamma<1$. Setting $\Lambda_{n}=\max \left(\lambda_{n}, \lambda_{n-1}\right)$, we get from (5.3) that

$$
\Lambda_{n+1} \leq \sqrt{\gamma} \Lambda_{n}
$$

So, once $\lambda_{n}$ become small, they start to decrease exponentially. It follows that they exponentially decrease forever. 
The final remark: since the constants in the Schwarz lemma and the Koebe principle depend only on $\|f\|$, the constants $\rho$ and $L$ depend only on this data as well. Moreover, all estimates are uniform in the case of negative Schwarzian derivative.

Recall that a one-dimensional homeomorphism $h$ is called quasi-symmetric if any two adjacent commensurable intervals $I$ and $J$ are mapped into those commensurable:

$$
\frac{|I|}{|J|} \leq K \Rightarrow \frac{|f I|}{|f J|} \leq \gamma(K)
$$

Denote by $\mathscr{F}^{0}$ the set of Fibonacci maps for which inf $\lambda_{n}=0$.

Lemma 5.3. (1) The set $\mathscr{F}^{0}$ is invariant under quasi-symmetric conjugacy.

(2) The set $\mathscr{F}^{0}$ is $C^{0}$-open in the $C^{2}$-balls $B(r)$ of the space $\mathscr{F}$.

Proof. The first point is clear from the definitions. The second follows from the fact that the constants in the previous lemma are uniform over $B(r)$.

Let us write $\alpha_{n} \sim \beta_{n}$ if $\left|\log \left(\alpha_{n} / \beta_{n}\right)\right|$ exponentially decrease and $\alpha_{n} \asymp \beta_{n}$ if it is bounded.

The next lemma gives the asymptotical formula of Theorem 1.3(1) for the subclass $\mathscr{F}^{0}$ (compare Tangerman and Veerman [TV]).

Lemma 5.4. For any $f \in \mathscr{F}^{0}$ the following asymptotical formulas hold:

(1) $\lambda_{n+1} \sim \lambda_{n} / \sqrt[3]{2}$.

(2) $\lambda_{n} \sim a 2^{-n / 3}$.

(3) $d_{n} \sim(1 / 2)^{n^{2} / 6+\beta n+\gamma}$

for some constants $a>0, \beta$, and $\gamma$. Moreover,

$$
\left|\log \left(a / \lambda_{0}\right)\right| \leq R(\|f\|),
$$

and the constant $R$ is uniform over maps with negative Schwarzian derivative. Proof. Since $\lambda_{n}$ exponentially decrease, Lemma 5.1 yields for $x \in I_{1}^{n+1}$

$$
\left|\left(f^{u(n)-1}\right)^{\prime}(x)\right| \sim d_{n} / d_{n+1}^{2} .
$$

Substituting this into the recurrent equation (5.1), we get

$$
\lambda_{n+1}^{2} \sim \frac{1}{2} \lambda_{n} \lambda_{n-1}
$$

Setting $s_{n}=\log \left(\lambda_{n} / \lambda_{n-1}\right)$, we have from the last formula

$$
s_{n+1}=-\frac{1}{2} s_{n}-\frac{1}{2} \log 2+O\left(q^{n}\right)
$$

with $q<1$. It yields

$$
s_{n}=-\frac{1}{3} \log 2+O\left(\rho^{n}\right)
$$

with $\rho=\max \left(\frac{1}{2}, q\right)$, which proves the first point of the lemma. 
Setting now $c=\frac{1}{3} \log 2, \nu_{n}=\log \lambda_{n}+c n$ we get from $(5.7)$

$$
\nu_{n+1}=\nu_{n}+O\left(\rho^{n}\right) \text {. }
$$

So, there is a limit

$$
\lim \nu_{n} \equiv \log a=\nu_{0}+O(1),
$$

with exponential convergence and the constant depending only on $\|f\|$ and uniform over maps with negative Schwarzian. Equivalently

$$
a \equiv \lim \lambda_{n} e^{n c} \asymp \lambda_{0} .
$$

It proves the second point together with the last remark. The reader can easily derive the third point from the second one.

Let us estimate now the ratio of any two intervals $M_{s_{1} \cdots s_{n}}^{n} \subset M_{s_{1} \cdots s_{n-1}}^{n-1}$. The previous lemma gives the asymptotics for the ratio $\lambda_{n} \equiv\left|M_{0 \cdots 0}^{n}\right| /\left|M_{0 \cdots 0}^{n-1}\right|$. Besides, $M_{s_{1} \cdots 10}^{n}=M_{s_{1} \cdots 1}^{n-1}$. Other cases are covered by the following lemma.

Lemma 5.5. For $f \in \mathscr{F}^{0}$ the following scaling laws hold:

$$
\frac{\left|M_{0 \cdots 01}^{n}\right|}{\left|M_{0 \cdots 0}^{n-1}\right|} \equiv \frac{\left|J^{n}\right|}{\left|I^{n-1}\right|} \sim \frac{a^{2}}{2^{2(n+1) / 3}} .
$$

If $\left[s_{1} \cdots s_{n-1}\right] \neq[0 \cdots 0]$ then

$$
\frac{\left|M_{s_{1} \cdots s_{n-1} 1}^{n}\right|}{\left|M_{s_{1} \cdots s_{n-1}}^{n-1}\right|} \sim \frac{a^{2}}{2^{2(n-1) / 3}}
$$

and

$$
\frac{\left|M_{s_{1} \cdots s_{n-1} 0}^{n}\right|}{\left|M_{s_{1} \cdots s_{n-1}}^{n-1}\right|} \sim \frac{a^{2}}{2^{2(n-2) / 3}}
$$

where $a$ is the constant from Lemma 5.4. All asymptotics are uniformly exponential.

Proof. Let us consider a chain of two maps

$$
\left(I^{n-1}, J^{n}\right) \underset{f}{\rightarrow}\left(I_{1}^{n-1}, J_{1}^{n}\right) \underset{f^{u(n-2)-1}}{\rightarrow}\left(I^{n-2}, I^{n}\right) .
$$

Note that by Lemma $5.4\left|I^{n}\right| \sim\left|x_{u(n)}\right|$. Setting $r_{n}=\left|J^{n}\right| /\left|I^{n-1}\right|$ we get

$$
\frac{\left|f J^{n}\right|}{\left|f I^{n-1}\right|} \sim 1-\left(1-r_{n}\right)^{2} \sim 2 r_{n} .
$$

On the other hand, $f^{u(n-2)-1}$ has an exponentially small distortion on $I_{1}^{n-1}$, and hence

$$
2 r_{n} \sim \frac{\left|I^{n}\right|}{\left|I^{n-2}\right|} \sim \lambda_{n} \lambda_{n-1} \sim \frac{a^{2}}{2^{(2 n-1) / 3}},
$$

and the first asymptotical formula is proved. 
In order to get the others, consider the map

$$
f^{k}: M_{s_{1} \cdots s_{n-1}}^{n-1} \rightarrow I^{n-2}
$$

for an appropriate $k$. It carries $M_{s_{1} \cdots s_{n-1} 0}^{n}$ into $J^{n-1}$ and $M_{s_{1} \cdots s_{n-1}}^{n}$ into $I^{n}$ with exponentially small distortion. It yields the result.

Now we can prove the next piece of Theorem 1.3 for $f \in \mathscr{F}^{0}$.

Lemma 5.6. For $f \in \mathscr{F}^{0}$ the critical orbit closure $\overline{\mathscr{O}}$ has Hausdorff dimension 0 .

Proof. Let us consider covering of $\overline{\mathscr{O}}$ by the intervals $M_{s_{1} \cdots s_{n}}^{n}$. By the above two lemmas, the lengths of these intervals decrease uniformly superexponential $\left(O\left(q^{n}\right)\right.$ for any $\left.q \in(0,1)\right)$, while their number increases exponentially $\left(\leq 2^{n}\right)$. Let $\gamma=-\log 2 / \log q ; l_{\gamma}$ be the Hausdorff measure on $\overline{\mathscr{O}}$ of exponent $\gamma$. Then

$$
l_{\gamma}(\overline{\mathscr{O}}) \leq C 2^{n} q^{n \gamma} \leq C .
$$

Hence, $\operatorname{dim} \overline{\mathscr{O}} \leq \gamma$, and $\gamma$ is arbitrary small positive number.

Now we are going to show that the geometry of the set $\overline{\mathscr{O}}$ is completely determined by only one parameter $a$ from Lemma 5.4. Let $f$ and $g$ be two Fibonacci maps,

$$
\phi: \overline{\mathscr{O}}(f) \rightarrow \overline{\mathscr{O}}(g)
$$

be the natural topological conjugacy. Let us say that $\phi$ is smooth if for any $x \in \overline{\mathscr{O}}$ there exist

$$
\lim \frac{|\phi(x)-\phi(y)|}{|x-y|} \neq 0
$$

as $y \rightarrow x$ along $\overline{\mathscr{O}}(f)$, and this limit depends continuously on $x$.

Lemma 5.7. If two Fibonacci maps $f$ and $g$ in $\mathscr{F}^{0}$ have the same parameter $a$, then the conjugacy $\phi$ is smooth on $\overline{\mathscr{O}}(f)$.

Proof. Indeed, it follows from Lemmas 5.4 and 5.5 that for any Fibonacci sequence $\bar{s}=s_{0} s_{1} \cdots$ there is a limit

$$
\lim _{n \rightarrow \infty} \frac{\left|M_{s_{1} \cdots s_{n}}^{n}(f)\right|}{\left|M_{s_{1} \cdots s_{n}}^{n}(g)\right|}
$$

depending continuously on $\bar{S}$.

Lemma 5.8. Let $f \in \mathscr{F}^{0}, n=\left[s_{1} \cdots s_{k}\right]$ be the Fibonacci expansion of $n$. Then

$$
\left|\left(f^{n}\right)^{\prime}\left(x_{1}\right)\right| \sim 2^{(2 / 3) \sum m s_{m}+\gamma \sum s_{m}+\delta}
$$

for some constants $\gamma$ and $\delta$.

Proof. Let $m_{i}$ be the places where $s_{m_{i}}=1$. Decompose the $n$-orbit of $x_{1}$ into the parts of length $u\left(m_{i}\right)$. By (5.5) it gives the factorization of the derivative into factors of order $\sim 2 \lambda_{m_{i}+1}^{-2}$. Now Lemma 5.4 implies the required asymptotics. 
Clearly, it follows from the last lemma that the growth of the $n$-fold derivative at $x_{1}$ is subexponential. The maximal growth of order $\exp \kappa(\log n)^{2}$ (which is faster than any power $n^{\gamma}$ ) is attained at moments $u(m)-1$. However, at the next moments $n=u(m)$ the derivative drops to $n^{\gamma}$ with $\gamma=$ $2 \log 2 / 3 \log \left(\frac{\sqrt{5}+1}{2}\right)<1$. These oscillations are balanced in a "convergent way".

Lemma 5.9. The series

$$
\sum_{n=1}^{\infty} \frac{1}{\left|\left(f^{n}\right)^{\prime}\left(x_{1}\right)\right|^{\alpha}}
$$

is convergent for any $\alpha>0$.

Proof. By the last lemma, this series has a majorant of the form

$$
\sum_{s_{m} \in\{0,1\}} 2^{-\sum_{m=1}^{k}(a m+b) s_{m}}=\prod_{m=1}^{\infty}\left(1+\frac{1}{2^{a m+b}}\right)<\infty .
$$

This lemma and the Nowicki-van Strien Theorem [NvS] imply the existence of an ahsolutely continuous invariant measure for $f \in \mathscr{F}^{0}$. So, Theorem 1.3 is proved for the subclass $\mathscr{F}^{0}$.

\section{ReAl RENORMALizations}

Now we need another class of maps on which we can define a renormalization in such a way that the Fibonacci maps can exactly be characterized as infinitely renormalizable. Let

$$
J=[a, b], \quad T=[\alpha, \beta], \quad \text { where }-1<a<b<\alpha<\beta<1,
$$

$\operatorname{Dom}(f)=J \cup T$, and let $f: \operatorname{Dom}(f) \rightarrow[-1,1]$ be a $C^{2}$-smooth map such that (see Figure 2)

(i) $f \mid J$ is a diffeomorphism from $J$ onto $[-1,1]$, which may be either orientation preserving or orientation reversing.

(ii) $f \mid T$ is a unimodal map from $T$ into $[-1,1]$ with nondegenerate minimum point $x_{0}=0$ and with $f(\partial T)=1$.

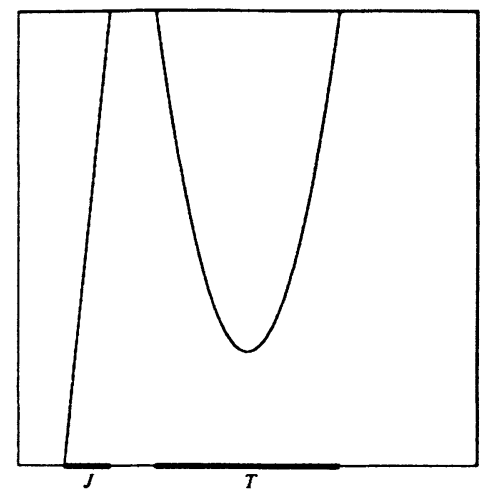

Figure 2. Graph of a function in $\mathscr{A}_{0}^{+}$. 
Let us denote the space of all such maps by $\mathscr{A}$. Since we do not specify whether $f \mid J$ preserves or reverses orientation, $\mathscr{A}$ can be decomposed into the union of two connected components $\mathscr{A}^{+}$and $\mathscr{A}^{-}$, where "+" corresponds to the case of orientation-preserving $f \mid J$.

Now suppose that some map $f \in \mathscr{A}$, with critical point $x_{0} \in T$, satisfies the conditions that the critical value $x_{1}=f\left(x_{0}\right)$ lies in $J$ and that its image $x_{2}=f\left(x_{1}\right)$ lies back in $T$. Then we will be interested in two segments of the first return map from $T$ to itself, as follows. There is an interval $T_{1}$ around the critical point which is mapped unimodally by $f^{2}$ into $T$, with both end points of $T_{1}$ mapping to one end point of $T$. Further, there is a disjoint interval $J_{1} \subset T$ which maps diffeomorphically onto $T$ under the map $f$ itself. Here we choose $J_{1}$ to the left of $T_{1}$ if $f \mid J$ preserves orientation, or to the right of $T_{1}$ if $f \mid J$ reverses orientation (so that $J_{1}$ lies on the same side of 0 as $x_{2}$ ). The resulting map $V f: J_{1} \cup T_{1} \rightarrow T$, affinely conjugated (rescaled) so that $T$ is replaced by the original interval $[-1,1]$, is the required renormalization $R f$ (there is choice of two rescalings; select that one which makes the critical point to be the minimum point). This renormalization interchanges the two spaces $\mathscr{A}^{+}$and $\mathscr{A}^{-}$. If $f$ is $n$-fold renormalizable, then $R^{n} f$ comes as rescaling of a map $V^{n} f \equiv f_{n}$, the restriction of appropriate iterates of $f$ to the union of two appropriate intervals, $T_{n}$ and $J_{n}$.

Let $T_{+}$and $T_{-}$be the semi-intervals on which 0 divides $T$. The kneading sequence of $f \in \mathscr{A}$ is the sequence of symbols $U_{n} \in\left\{T_{+}, T_{-}, J\right\}$ such that $x_{n} \equiv f^{n} 0 \in U_{n}$. Two maps $f \in \mathscr{A}^{+}$(or $\mathscr{A}^{-}$) with nonescaping critical point and without limit cycles are topologically conjugate if and only if they have the same kneading sequence (compare [MT]).

In terms of kneading sequences the above renormalization can be described in the following way. The renormalizable kneading sequences start with $J T_{s}$, $s \in\{+,-\}$. To write its renormalization perform the following operations moving along the sequence

(i) When you see $J$, cross it;

(ii) When you see $T_{s} J, s \in\{+,-\}$, change $T_{s}$ for $T_{k s}$ provided $f \in \mathscr{A}^{k}$, $k \in\{+,-\}$;

(iii) When you see $T_{s} T_{r}$, change the first $T_{s}$ for $J$.

Let us say that a map $f \in \mathscr{A}^{+}$is a Fibonacci map if it has the following kneading sequence:

$$
\mathrm{fib}^{+}=J\left|T_{-}\right| T_{+}\left|J T_{+}\right| J T_{-} T_{-}\left|J T_{-} T_{+} J T_{-}\right| \cdots .
$$

(In order to write the block from $u(n)+1$ to $u(n+1)$, repeat the beginning of the sequence until the moment $u(n-1)$ and then change the last symbol $T_{s}$ for the "opposite" one, $T_{-s}$.) Denote this class of maps by $\mathscr{F}^{+}$. Similarly, the kneading sequence of a map $f \in \mathscr{F}^{-}$is produced by the same rule but with different initial:

$$
\mathrm{fib}^{-}=J\left|T_{+}\right| T_{+}\left|J T_{-}\right| J T_{+} T_{-}\left|J T_{+} T_{+} J T_{+}\right| \cdots .
$$

A class $\mathscr{F}$ of Fibonacci maps is defined as $\mathscr{F}^{+} \cup \mathscr{F}^{-}$. One can also describe 
this class by the following properties: $x_{1} \in J$, and $f^{u(n-1)}$ is well defined and monotone on the interval $\left[\left[0, x_{u(n)}\right]\right]$, and

$$
f^{u(n-1)}\left(\left(0, x_{u(n)}\right)\right) \equiv\left(\left(x_{u(n-1)}, x_{u(n+1)}\right)\right) \ni 0 .
$$

If we want to emphasize that $f \in \mathscr{A}$, then we say that $f$ has type $(2,1)$. In the unimodal case we say that $f$ is of type (2) (see the next section for more general discussion). As in the unimodal case, we will use the notation $T^{n}$ and $J^{n}$ for the intervals $\left[\left[x_{u(n)}, x_{u(n)}^{\prime}\right]\right]$ and $\left[\left[x_{u(n-1)}, x_{u(n-1)+u(n+1)}\right]\right]$ correspondingly (do not confuse with $T_{n}$ and $J_{n}$ introduced above).

Lemma 6.1. A map $f \in \mathscr{A}$ is infinitely renormalizable if and only if it is a Fibonacci map: $f \in \mathscr{F}$. In this case the following inclusions hold:

$$
\begin{gathered}
T^{n+2} \subset T_{n} \subset T^{n+1}, \\
J^{n+2} \subset J_{n} .
\end{gathered}
$$

Proof. Let $f \in \mathscr{A}$ be infinitely renormalizable. Then one can check by induction that

$$
f_{n} \mid T_{n}=f^{u(n+1)} \quad \text { and } \quad f_{n} \mid J_{n}=f^{u(n)} .
$$

Since $f_{n-1}$ is renormalizable,

$$
x_{u(n)}=f_{n-1}(0) \in J_{n-1} \quad \text { and } \quad x_{u(n+1)}=f_{n}(0) \in T_{n-1} .
$$

Hence, $x_{u(n+1)}$ lies closer to 0 than $x_{u(n)}, n=1,2, \ldots$.

Let us study now the combinatorics of several first iterates of 0 . Since $f$ is renormalizable,

$$
T^{2} \equiv\left[\left[x_{2}, x_{2}^{\prime}\right]\right] \subset T \subset\left[x_{1}, x_{1}^{\prime}\right] \equiv T^{1} .
$$

Furthermore, $x_{3}=f_{2}(0) \in T_{1}$; hence, $x_{4}=f x_{3} \in J$. So,

$$
J^{2} \equiv\left[x_{1}, x_{4}\right] \subset J .
$$

Consider now the map $\sigma: \mathbf{N} \rightarrow \mathbf{N}$ of the set of natural numbers: if $m=$ $\sum u\left(l_{i}\right)$ is the Fibonacci expansion of $m$ then $\sigma(m)=\sum u\left(l_{i}+1\right)(\sigma$ is induced by the shift on the space of Fibonacci expansions). Then we have the following rule:

$$
\left(f_{n}\right)^{m}(0)=x_{\sigma^{n}(m)} .
$$

So, if we have a combinatorial property of several points $x_{m}$, then replacing $f$ by $f_{n}$ we immediately get the same property of points $x_{\sigma^{n} m}$ (provided $f$ is infinitely renormalizable). In particular, we can replace points $x_{1}, x_{2}, x_{4}$ in (6.4) and (6.5) by $x_{u(n+1)}, x_{u(n+2)}, x_{u(n+1)+u(n+3)}$. Then we obtain the required properties (6.1) and (6.2).

Let us show now that $x_{1}$ and $x_{2}$ lie on the same side of 0 for $f \in \mathscr{A}^{+}$, and they lie on the opposite sides of 0 for $f \in \mathscr{A}^{-}$. Indeed, otherwise consider $f \mid\left[x_{1}, x_{4}\right]$ and conclude that $x_{5}$ lies farther from 0 than $x_{2}$. 
Changing $f$ for $f_{1}$ we get the same statement for the points $x_{2}$ and $x_{3}$. Since the renormalization interchanges $\mathscr{A}^{+}$and $\mathscr{A}^{-}$, we conclude that $\left(\left(x_{1}, x_{3}\right)\right) \ni 0$. Replacing $f$ by $f_{n-2}$ we get $(6.0)$.

Finally, since $x_{2} \in T, f \mid\left[0, x_{2}\right]$ is well defined and monotone. Replacing it again by $f_{n-2}$ we conclude that $f^{u(n-1)} \mid\left[0, x_{u(n)}\right]$ is well defined and monotone. So, $f$ is a Fibonacci map.

Vice versa, let $\mathrm{fib}_{n}^{s}, s \in\{+,-\}$, be the initial parts of length $u(n)$ of the kneading sequences $\mathrm{fib}^{s}$. Then one can easily check by induction that the renormalization turns $\mathrm{fib}_{n}^{s}$ into $\mathrm{fib}_{n-1}^{-s}$. So, it interchanges $\mathrm{fib}^{s}$ and $\mathrm{fib}^{-s}$, which certainly implies that both sequences are infinitely renormalizable.

Now let us briefly discuss topology on the space $\mathscr{A}$ (compare $\S 4)$. We can restrict ourselves to the subspace $\mathscr{A}_{0} \subset \mathscr{A}$ consisting of those $f$ for which $f \mid T$ is an even function, $f(-x)=f(x)$. Then we can write $f \mid T$ uniquely as

$$
f(x)=A_{x_{1}} \circ f_{T} \circ Q \circ A_{T}
$$

where $A_{T}$ is the orientation-preserving linear map which carries $T$ onto $[-1,1], Q$ is the squaring map $\xi \mapsto \xi^{2}, f_{T}$ is some orientation-preserving diffeomorphism of $[0,1]$, and $A_{x_{1}}$ is the orientation-preserving affine map which carries $[0,1]$ onto $\left[x_{1}, 1\right]$, where $x_{1}=f(0)$ is the critical value. Similarly, we can write $f \mid J$ as $f_{J} \circ A_{J}$ where $A_{J}$ is the orientation-preserving affine map from $J$ onto $[-1,1]$ and where $f_{J}$ is a diffeomorphism of $[-1,1]$.

Now we suppose that both $f_{J}$ and $f_{T}$ are $C^{2}$-smooth. The $C^{k}$-topology on $\mathscr{A}_{0}, k \leq 2$, comes from the $C^{k}$-topology on the space of diffeomorphisms $f_{T}$ and $f_{J}$, together with the Euclidean topology on the finite-dimensional space of parameters $a, b, \alpha, \beta, x_{1}$. Let $\|f\|$ denote the maximum of the $C^{2}$-norms for $f_{J}, f_{J}^{-1}$ and $f_{T}, f_{T}^{-1}$ which is a continuous functional on our space.

We can assume without loss of generality that the original map $f$ is quadratic near 0 (though this property is not preserved under renormalization). Let us remark also that clearly all estimates of $\S \S 4,5$ hold not only for unimodal maps but in the class $\mathscr{A}$ as well.

Lemma 6.2. The norms $\left\|R^{n} f\right\|$ are uniformly bounded.

Proof. By (6.3), $f_{n} \mid T_{n}=f^{u(n+1)}$ which can be decomposed as a quadratic map and the diffeomorphism

$$
f^{u(n+1)-1}: H^{n+2} \rightarrow T^{n-1}
$$

(see Lemma 4.1). On the other hand,

$$
f^{u(n+1)-1}\left(f T_{n}\right)=f_{n} T_{n} \subset T_{n-1} \subset T^{n}
$$

(the last inclusion is by (6.1)). It follows from (6.6), (6.7), and a priori bounds proven in $\S 4$ that $f^{u(n+1)-1} \mid f T_{n}$ has bounded distortion. By rescaling we get

$$
\log \left|\frac{\left(R^{n} f\right)_{T}^{\prime}(x)}{\left(R^{n} f\right)_{T}^{\prime}(y)}\right|=O(|x-y|)
$$


for any $x, y \in[0,1]$. This implies

$$
\left|\frac{\left(R^{n} f\right)_{T}^{\prime \prime}}{\left(R^{n} f\right)_{T}^{\prime}}\right|=O(1) .
$$

Because of bounded distortion, the derivative $\left(R^{n} f\right)_{T}^{\prime}$ is uniformly bounded from below and above, and the boundedness property for the second derivative $\left(R^{n} f\right)_{T}^{\prime \prime}$ follows. The same argument applies to $\left(R^{n} f\right)_{J}$ and to the inverse maps.

Corollary 6.3. If inf $\lambda_{n}>0$ then there is a $C^{1}$-convergent sequence of renormalizations $R^{n_{i}} \rightarrow g \in \mathscr{A}$.

Proof. It follows from the assumption and inclusions (6.1) that the ratio $\left|T_{n}\right|:\left|T_{n-1}\right|$ is bounded away from 0. Moreover, Lemma 4.9 and (6.2) imply the same for the ratio $\left|J_{n}\right|:\left|T_{n-1}\right|$. Now one can play the "distortion game" in manner of $\S 4$ to check that three complementary gaps (that is, components of $\left.T_{n-1} \backslash\left(T_{n} \cup J_{n}\right)\right)$ are also commensurable with $T_{n-1}$. After rescaling we conclude that the domains $\operatorname{Dom}\left(R^{n} f\right)$ do not degenerate, so we can select a convergent sequence $\operatorname{Dom}\left(R^{n_{i}} f\right)$. Then by the last lemma, families of diffeomorphisms $\left\{\left(R^{n_{i}} f\right)_{T}\right\}$ and $\left\{\left(R^{n_{i}} f\right)_{J}\right\}$ are $C^{1}$-precompact, and we can extract from them convergent subsequences as well.

For an interval $I \subset \mathbf{R}$ denote by $P(I)$ the plane slitted along two rays

$$
P(I)=\mathbf{C} \backslash(\mathbf{R} \backslash I) .
$$

Let us introduce now a subspace $\mathscr{E} \subset \mathscr{A}$ consisting of maps $f: T \cup J \rightarrow$ $[-1,1]$ with the following property: The map $f_{T}^{-1}:[0,1] \rightarrow[0,1]$ can be analytically continued to a map $P[0,1] \rightarrow P[0,1]$, and $f^{-1}:[-1,1] \rightarrow J$ can be analytically continued to a map $P[-1,1] \rightarrow P(J)$.

Lemma 6.4. Let $R^{n_{i}} f \rightarrow g$ in $C^{1}$-topology. Then the limiting function $g$ belongs to the class $\mathscr{E}$.

Proof. The map $\left(R^{n} f\right)_{T}^{-1}$ can be written as long compositions of type $h_{1} \circ q_{1} \circ$ $\cdots h_{k} \circ q_{k}$ where $h_{i}$ are diffeomorphisms between appropriate intervals with a small total distortion while $q_{i}$ are square root maps (we reserve this term for affine conjugates to the standard square root). Such a map can be rewritten as $H_{n} \circ Q_{n}$ where the distortion of $H_{n}$ does not exceed the total distortion of $h_{i}$, $i=1, \ldots, n$, and $Q_{n}$ is the composition of $Q_{i}$ renormalized by appropriate Möbius maps (see $[\mathrm{S}, \mathrm{Sw} 2])$. The maps $Q_{n}$ analytically map $P[-1,1]$ into itself and hence form a normal family. So, we can select a convergent sequence $Q_{n} \rightarrow Q$ with $Q$ to be a selfmap of $P[-1,1]$. On the other hand, $H_{n} \rightarrow$ id in $C^{1}$-topology. So, $\left(g_{T}\right)^{-1}=Q$. In the same way we can treat $g_{J}$.

Correspondence between Fibonacci maps of classes $\mathscr{U}$ and $\mathscr{A}^{-}$. We are going to describe an easy surgery interchanging these classes without touching the critical orbit. It will follow that any result about the critical orbit established in one of the classes immediately yields the same statement in the other class. 
Let $f \in \mathscr{U}$ be a unimodal Fibonacci map. Let us restrict it onto the union of two disjoint intervals

$$
I^{2} \cup J^{2} \equiv\left[x_{5}, x_{2}\right] \cup\left[x_{1}, x_{4}\right] .
$$

Then let us embed these intervals into disjoint intervals $T$ and $J$ correspondingly and continue $f$ to a map of class $\mathscr{A}^{-}$defined on $T \cup J$.

Vice versa, given a Fibonacci map $g \in \mathscr{A}^{-}$, we can also restrict it onto the union (6.8) and then continue to a unimodal map of class $\mathscr{U}$. This is possible since $g\left(x_{5}\right) \equiv x_{6}<x_{5} \equiv g\left(x_{4}\right)$.

Since orb $(0) \subset I^{2} \cup J^{2}$, the above surgeries keep the critical orbit untouched.

Remark 6.1. We would like to thank E. Aurell and M. Zaks who have recently informed us of the paper [KP] which contains a computer-assisted attempt to apply renorm-group method to the Fibonacci map. Unlike [KP], our approach does not destroy the dynamics.

\section{PolyNOMIAL-LIKE MAPS}

Now we are going to show that all polynomial-like maps $f \in \mathscr{A}^{-}$(or $\mathscr{A}^{+}$) are quasi-symmetrically conjugate. It is convenient to introduce more general terminology.

Consider $k+1$ topological disks $U_{i}$ and $V$ bounded by piecewise smooth curves, and such that $\mathrm{cl} U_{i}$ are disjoint and contained in $V$. Let us say that

$$
f: \bigcup U_{i} \rightarrow V
$$

is a polynomial-like map of type $\left(n_{1}, \ldots, n_{k}\right)$ if $f \mid U_{i}$ is a branched covering of degree $n_{i} ; d=\sum n_{i}$ is called the degree of $f$. Note that polynomial-like maps of type $(d)$ are exactly polynomial-like maps in the sense of Douady and Hubbard [DH].

Lemma 7.1. Any polynomial-like map $f: U_{1} \cup U_{2} \rightarrow V$ of type $(2,1)$ is quasiconformally conjugate to a cubic polynomial with at least one escaping critical point.

Proof. Consider an "eight-like" neighborhood $N$ of $U_{1} \cup U_{2}$ and smoothly continue $f$ there so that $f$ becomes a double covering on the annulus around $U_{1}$ and a diffeomorphism on the annulus around $U_{2^{i}}$, and both annuli are mapped on the same annulus around $V$ (see Figure 3, p. 452).

Then continue $f$ to a slightly bigger domain so that it turns into a threesheeted smooth covering of a topological disk over a bigger disk. Now use the Douady-Hubbard surgery $[\mathrm{DH}]$ in order to conjugate quasi-conformally this map to a cubic polynomial.

Lemma 7.2. Any polynomial-like map $f \in \mathscr{A}^{-}$is quasi-symmetrically conjugate to a real cubic polynomial with one escaping critical point.

Proof. For $f \in \mathscr{A}^{-}$one can carry out the above construction in an R-symmetrical way. 


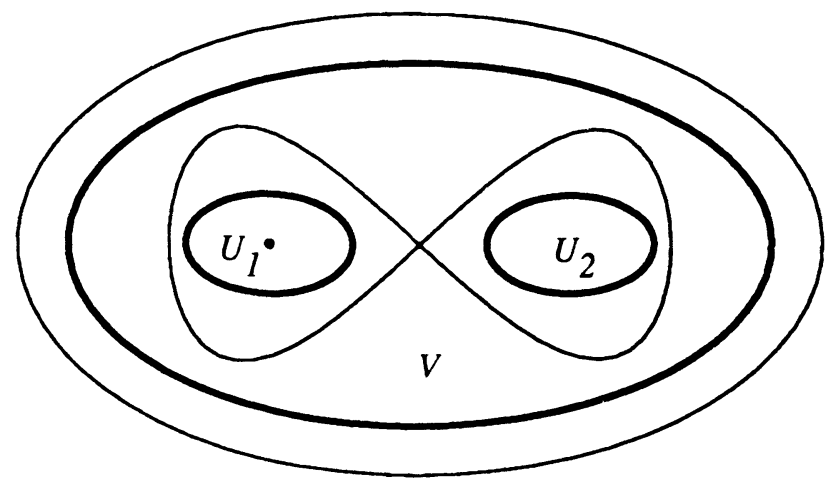

FIGURE 3

Lemma 7.3. All Fibonacci real cubic polynomials are quasi-symmetrically conjugate.

Proof. Consider a locus $F_{+}$of real cubic polynomials $z \mapsto z^{3}-3 a^{2} z+b$ for which the critical point $a$ is a preimage of the left fixed point (it is equivalent to $b=2 a^{3}-2 a$ ) and $a<\frac{1}{3}$. By Branner and Douady [BD], there is a natural one-to-one correspondence between $F_{+}$and the $\frac{1}{2}$-locus of quadratic polynomials $z \mapsto z^{2}-c$ with $-2 \leq c<-\frac{3}{4}$. Hence, in $F_{+}$there is only one Fibonacci map (Theorem 1.1). On the other hand, conjugacy classes of cubic maps with escaping critical point $a$ (which means $b<2 a^{3}-2 a$ ) are in one-toone correspondence with $F+$ as well: go toward the curve $b=2 a^{3}-2 a$ along external rays (this argument is due to Douady).

From the last two lemmas we have immediate

Corollary 7.4. All polynomial-like Fibonacci maps $f \in \mathscr{F}^{-}$are quasi-symmetrically conjugate.

Corollary 7.5. All polynomial-like Fibonacci maps belong either to the set $\mathscr{F}_{0}$ or to its complement.

Proof. For maps $f \in \mathscr{F}^{-}$this follows from Corollary 7.4 and Lemma 5.3. For maps $f \in \mathscr{F}^{+}$just observe that it belongs to $\mathscr{F}^{0}$ or its complement together with the renormalization.

Now we will give an example of a polynomial-like map belonging to $\mathscr{F}_{0}$ which will yield that all Fibonacci polynomial-like maps belong to $\mathscr{F}_{0}$.

Example 7.1. Consider disjoint union of two intervals $I=[-1, \lambda]$ and $J=$ $\left[-c,-c+q \lambda^{2}\right]$ with positive $c, q, \lambda, c$ is big, $\lambda$ is small. Let $f \mid I$ be a quadratic map $x \mapsto q x^{2}-c$, while $f \mid J$ be linear $x \mapsto \alpha x+b$.

Let us adjust parameters $\alpha, b, c, q, \lambda$ in such a way that

$$
0 \mapsto-c \rightarrow-1 \mapsto \lambda \mapsto-c+q \lambda^{2} \mapsto v \in[0, \lambda] .
$$

It yields the relations

$$
q=c+\lambda \sim c, \quad \alpha=\frac{1+v}{(c+\lambda) \lambda^{2}} \sim \frac{1}{c \lambda^{2}}, \quad b=\alpha c-1 \sim \frac{1}{\lambda^{2}} .
$$


There remain three free parameters $c, \lambda$, and $v$. Let us show that for $c^{2} \lambda^{2}<1$ this map is cubic-like. To this end consider a disk $D=\{z:|z|<2\}$. On its boundary $\partial D$ our map acts as

$$
f(z)=c\left(z^{2}-1\right)+\lambda z^{2} \sim \mathcal{c}\left(z^{2}-1\right)
$$

Hence,

$$
3 c<|f(z)|<5 c \text { for } z \in \partial D .
$$

Consider a disk $V=\{z:|z|<2 c\}$ and its inverse image $U_{1}$ (under the quadratic map.) By (7.2), $U_{1} \subset D$ and $f: U_{1} \rightarrow V$ is a quadratic-like map. Moreover, $U_{1} \supset[-1,1]$ since $f[-1,1]=[-c, \lambda] \subset V$.

Furthermore, consider the preimage $U_{2}$ of $V$ under the linear map $z \mapsto$ $\alpha z+b$. It is a disk containing $J$ of radius

$$
2 c / \alpha \sim 2 c^{2} \lambda^{2}<2
$$

(by (7.1)). Hence, for big enough $c$ the closure of this disk is contained in $V$ and does not intersect $\operatorname{cl} U_{1}$. So, $f: U_{1} \cup U_{2} \rightarrow V$ is a polynomial-like map.

Now one can adjust $v$ to get a Fibonacci map. Since $f$ has nonpositive Schwarzian derivative, it belongs to $\mathscr{F}^{0}$ provided $\lambda$ is sufficiently small (Lemma 5.2).

Renormalization of a quadratic-like Fibonacci map. This procedure associates to a quadratic-like Fibonacci map (of type (2)) a cubic-like Fibonacci map (of type $(2,1))$. It will complete the proof of Theorem 1.3 for quadratic-like Fibonacci maps (in particular, for the quadratic polynomial). We can restrict ourselves to the case of the quadratic Fibonacci polynomial. Now let us consider the beginning of the Yoccoz partition construction (see [H]). Draw a curve $S$ consisting of two external rays through the fixed point $\alpha$ and an equipotential level $\gamma$. We will obtain two pieces of level 0 , namely, $W^{0}$ (containing 0 ) and $W_{1}^{0}$ (containing $x_{1}$ ). Define pieces of level $n$ as $n$-fold preimages of the pieces of level 0 . Denote by $W^{n}(x)$ the piece of level $n$ containing $x$; set $W^{n} \equiv W^{n}(0)$. Let us consider the piece $V \equiv W^{4} \supset T^{4}$ satisfying the property that

$$
\operatorname{cl} W^{4} \subset W^{3} \text {. }
$$

Define a piece $U_{1} \equiv W^{9} \supset T^{5}$ as the pull-back of $V$ of order 5 and $U_{2} \supset J^{5}$ as the pull-back of $V$ of order 3. One can check that $\operatorname{cl} U_{1}$ and $\operatorname{cl} U_{2}$ are pairwise disjoint and are contained in $V$ (it is a formal corollary from (7.3)). So, the map $g$ defined as $f^{5} \mid U_{1}$ and $f^{3} \mid U_{2}$ is polynomial-like of type $(2,1)$.

Remark 7.1. The above construction actually can be applied to any noninfinitely renormalizable "persistently recurrent" quadratic polynomial (see [L2]).

Geometry of $\omega(c)$ is not rigid. We would like to show that parameter a can really be changed in class $\mathscr{U}$, so the geometry of $\omega(c)$ is not rigid. The above example provides us with a Fibonacci map of class $\mathscr{A}$ with arbitrary small $\lambda_{0}=1 / c$. By Lemma 5.4, parameter $a \asymp \lambda_{0}$ is getting arbitrarily small as well. Renormalizing $f$ if necessary we obtain a Fibonacci map of class $\mathscr{A}^{-}$ 
with arbitrarily small $a$. Now the surgery of $\S 6$ turns this map into a unimodal Fibonacci map with the same parameter $a$.

Remark 7.2. Actually, in order to vary parameter $a$ in class $\mathscr{A}$ it is enough to observe that the renormalization turns $a$ into $a / \sqrt[3]{2}$.

\section{Polynomial-Like Property of ANAlytic FibonacCi MAPS}

In this section we will prove that analytic Fibonacci maps $f \in \mathscr{E}$ become polynomial-like after appropriate renormalization. Together with the results of the previous two sections it will complete the proof of Theorem 1.3.

For an interval $I \subset \mathbf{R}$ denote by $D(I)$ the Euclidean disk based upon $I$ as the diameter.

Lemma 8.1 (see $[\mathrm{S}]$ ). Let $\phi: P(I) \rightarrow P(J)$ be an analytic map which maps $I$ diffeomorphically onto $J$. Then $\phi D(I) \subset D(J)$.

Proof. The interval $I$ is a Poincare geodesic in $P(I)$, and the disk $D(I)$ is its Poincare neighborhood (of radius independent of $I$ ). Since $\phi$ contracts the Poincare metric, we have the required.

Lemma 8.2. Let $f \in \mathscr{E}$ be an analytic Fibonacci map. Given $n$, consider a disk $V=D\left(T_{n}\right)$ and its pull-backs $U_{1} \supset T_{n+1}$ and $U_{2} \supset J_{n+1}$ of order $u(n+2)$ and $u(n+1)$ correspondingly. Then $\mathrm{cl} U_{i}$ are disjoint and are contained in $V$.

Proof. Let $T_{n}=\left[\left[t_{n}, t_{n}^{\prime}\right]\right]$ with $t_{n}$ being closer to $x_{u(n+2)}$.

The branch $\phi: V \rightarrow U_{2}$ of $f^{-u(n+1)}$ satisfies the assumptions of Lemma 8.1, and hence $U_{2} \subset D\left(J_{n+1}\right)$. By the same reason, $f U_{1} \subset D(Q)$ where $Q \equiv$ $[b, a] \ni x_{1}$ is the monotone pull-back of $T_{n}$ of order $u(n+2)-1\left(b<x_{1}\right.$ is the preimage of $t_{n}$ ).

Now let $X_{n-1}$ be the component of $T_{n-1} \backslash T_{n}$ adjacent to $t_{n}$. Since $\sum\left|X_{n}\right|<$ $\infty$, we can select such an $n$ that

$$
\left|X_{n}\right|<\left|X_{n-1}\right| \text {. }
$$

By Lemma 4.1, the map $f^{u(n+2)-1}$ has a monotone continuation beyond the point $b$ to the interval $W$ which is mapped onto $X_{n-1}$. So, we have a threeinterval map

$$
f^{u(n+2)-1}: W \cup\left[b, x_{1}\right] \cup\left[x_{1}, a\right] \rightarrow X_{n-1} \cup\left[\left[t_{n}, x_{u(n+2)}\right]\right] \cup\left[\left[x_{u(n+2)}, t_{n}^{\prime}\right]\right] .
$$

Let $q=\left|x_{u(n+2)}\right|:\left|t_{n}\right|$. Applying the Schwarz lemma to (8.2) taking into account (8.1) we get

$$
\log \frac{a-b}{a-x_{1}} \leq \log 2+\log \frac{2}{1+q}
$$

so that

$$
\frac{x_{1}-b}{a-x_{1}} \leq \frac{3-q}{1+q}
$$

Now let us take the $f$-preimage of $D(Q)$. Since $f^{-1}$ is just a square root $\psi: \zeta \mapsto \sqrt{\zeta-x_{1}}$ on $D(Q)$, this preimage is contained in a domain based upon 
$T_{n+1}$ with attitude

$$
\dot{h}=\left|t_{n+1}\right| \sqrt{\frac{x_{1}-b}{a-x_{1}}} \leq\left|t_{n+1}\right| \sqrt{\frac{3-q}{1+q}} \leq\left|t_{n+1}\right| / q<t_{n} .
$$

Moreover, this domain is contained in the disk centered at zero of radius $\max \left(t_{n+1}, h\right)<t_{n}$. So, $\operatorname{cl} U_{1} \subset V$.

Let us show now that $\operatorname{cl} U_{1} \cap \operatorname{cl} U_{2}=\varnothing$. If $a-x_{1} \geq x_{1}-b$ then $\psi D(Q) \subset$ $D\left(T_{n+1}\right)$, and the statement follows. Assume that $x_{1}-b>a-x_{1}$. Then one can check the following elementary fact about the square root map: $\psi D[b, a]$ is convex if and only if $x_{1}-b \leq 3\left(a-x_{1}\right)$. By (8.3), the last estimate holds, so $\psi D(Q)$ is convex. Hence, $\psi D(Q) \cap D\left(J_{n+1}\right)=\varnothing$, and we are done.

\section{ApPendiX. Schwarz lemma and Koebe Principle}

We refer the reader to [Y1, G2, Sw1, Sw2, MvS, S] for the following technical background.

Let us consider four points $a<b<c<d$ and two nested intervals $L=$ $[a, d]$ and $H=[b, c]$. The Poincare length of $H$ in $L$ is the logarithm of an appropriate cross-ratio

$$
[H: L]=\log \frac{(d-b)(c-a)}{(d-c)(b-a)} .
$$

Let $g:(L, H) \rightarrow\left(L^{\prime}, H^{\prime}\right)$ be a $C^{3}$ diffeomorphism, and let

$$
S g=\frac{g^{\prime \prime \prime}}{g^{\prime}}-\frac{3}{2}\left(\frac{g^{\prime \prime}}{g^{\prime}}\right)^{2}
$$

be its Schwarzian derivative.

Schwarz Lemma. If $g$ has nonnegative Schwarzian derivative, then it contracts Poincaré length $\left[H^{\prime}: L^{\prime}\right] \leq[H: L]$.

Koebe Principle. Let $g$ has nonnegative Schwarzian derivative. If $[H: L] \leq l$ then $\left|g^{\prime}(x) / g^{\prime}(y)\right| \leq K(l)$ for any $x, y \in H$. Moreover, $K(l)=1+O(l)$ as $l \rightarrow 0$.

One can essentially extend the range of applications of these results combining the Schwarzian derivative condition on some intervals with bounded nonlinearity on others. Let us consider a chain of (closed) interval diffeomorphisms

$$
I_{1} \rightarrow J_{1} \rightarrow \cdots \rightarrow I_{n} \rightarrow J_{n}
$$

where $g_{i}: I_{i} \rightarrow J_{i}$ have nonnegative Schwarzian derivative while $h_{i}: J_{i} \rightarrow I_{i+1}$ are just $C^{2}$ smooth. Set $F=h_{n} \circ g_{n} \circ \cdots \circ h_{1} \circ g_{1}$. Let $G_{i} \subset \operatorname{int} I_{i}$ and $H_{i} \subset$ int $J_{i}$ be closed subintervals related by diffeomorphisms.

Denote by $\mathbf{h}$ the family of maps $h_{i}$, by $\mathbf{I}$ the family of intervals $I_{i}$, etc. Let $\left\|h_{i}\right\|=\max \left|h^{\prime \prime}(x) / h^{\prime}(x)\right|,\|\mathbf{h}\|=\max \left\|h_{i}\right\|$ be the maximal nonlinearity of $\mathbf{h},|\mathbf{I}|=\sum\left|I_{i}\right|$ be the total length of $\mathbf{I}, l=\left[G_{1}: I_{1}\right]$. 
Schwarz Lemma (smooth version). Expansion of the Poincare length by the map $F$ is controlled by $\mathbf{h}$ in the manner

$$
\left[H_{n}: J_{n}\right] \leq l+O(|\mathbf{J}|)
$$

with the constant depending on $\|\mathbf{h}\|$.

Koebe Principle (smooth version). Distortion of $F \mid G_{1}$ can be estimated as

$$
\left|\frac{F^{\prime}(x)}{F^{\prime}(y)}\right| \leq K(l ;|\mathbf{h}|,|\mathbf{J}|)
$$

where $K=1+O(l+|\mathbf{J}|)$ as $|\mathbf{J}|, l \rightarrow 0$ with the constant depending on $|\mathbf{h}|$.

\section{ACKNOWLEDGMENT}

We want to thank Branner, Douady, Sullivan, and Tresser, for helpful conversations. We also profited from the discussions with the participants of the Stony Brook dynamical systems seminar, particularly: Brucks, Yu. Lyubich, Shishikura, Tangerman, and Veerman.

\section{REFERENCES}

[BD] B. Branner and A. Douady, Surgery on complex polynomials, preprint, Matematic Institut, Denmark, 1987-05.

[BH] B. Branner and J. H. Hubbard, The iteration of cubic polynomials, Part II: patterns and parapatterns, Acta Math. (to appear).

[BL1] A. Blokh and and M. Lyubich, Non-existence of wandering intervals and structure of topological attractors for one dimensional dynamical systems, Ergodic Theory Dynamical Systems 9 (1989), 751-758.

[BL2] _ Measurable dynamics of S-unimodal maps of the interval, Ann. Sci. Ecole Norm. Sup. (4) 24 (1991), 545-573.

[BL3] _- Measure and dimension of solenoidal attractors of one-dimensional dynamical systems, Comm. Math. Phys. 127 (1990), 573-583.

[DH] A. Douady and J. H. Hubbard, On the dynamics of polynomial-like maps, Ann. Sci. Ecole Norm. Sup. (4) 18 (1985), 287-343.

[G1] J. Guckenheimer, Sensitive dependence to initial conditions for one-dimensional maps, Comm. Math. Phys. 70 (1979), 133-160.

[G2] - Limit sets of $S$-unimodal maps with zero entropy, Comm. Math. Phys. 110, 655-659.

[H] J. H. Hubbard, according to J.-C. Yoccoz, Puzzles and quadratic tableaux, preprint, 1990.

[HK] F. Hofbauer and G. Keller, Some remarks on recent results about S-unimodal maps, Ann. Inst. H. Poincaré Phys. Théor. 53 (1990), 413-425.

[L1] M. Lyubich, Non-existence of wandering intervals and structure of topological attractors for one dimensional dynamical systems, Ergodic Theory Dynamical Systems 9 (1989), 737-750.

[L2] _ On the Lebesgue measure of the Julia set of a quadratic polynomial, preprint, IMS, $1991 / 10$.

[KP] J. Ketoja and O. Piirila, On the abnormality of the period doubling bifurcation, Phys. Lett. A 138 (1989), 488-492.

[M] J. Milnor, On the concept of attractor, Comm. Math. Phys. 99 (1985), 177-195; 102 (1985), 517-519.

[MT] J. Milnor and W. Thurston, On iterated maps of the interval, Dynamical Systems, Proc. U. Md., 1986-87, (J. Alexander, ed.), Lecture Notes in Math., vol. 1342, Springer, New York, 1988, pp. 465-563. 
[Ma] M. Martens, Cantor attractors of unimodal maps, Thesis, 1990.

[MvS] W. de Melo and S. van Strien, A structure theorem in one-dimensional dynamics, Ann. of Math. (2) 129 (1989), 519-546.

[NvS] T. Nowicki and S. van Strien, Invariant measures exist under a summability condition for unimodal maps, Invent. Math. 105 (1991), 123-136.

[PTT] I. Procaccia, S. Thomae, and C. Tresser, First return maps as a unified renormalization scheme for dynamical systems, Phys. Rev. A 35 (1987), 1884-1900.

[S] D. Sullivan, Bounds, quadratic differentials, and renormalization conjectures, preprint, 1990.

[Sh] K. Shibayama, Fibonacci sequence of stable periodic orbits for one-parameter families of $C^{1}$-unimodal mappings, preprint.

[Sw1] G. Swiatek, Rational rotation numbers for maps of the circle, Comm. Math. Phys. 119 (1988), 109-128.

[Sw2] _ Bounded distortion properties of one-dimensional maps, preprint, SUNY, Stony Brook, $1990 / 10$.

[T] I. Tsuda, On the abnormality of period doubling bifurcations, Progress Theor. Phys. 66 (1985), 1985-2002.

[TV] F. Tangerman and P. Veerman, Scaling in circle maps. I, Comm. Math. Phys. 134 (1990), 89-107.

[VL] A. M. Vershik and A. M. Livshitz, Adic models of ergodic transformations, preprint, University of California, Berkeley, PAM-499 (1990).

[Y1] J.-C. Yoccoz, Il n'y a pas de contre-exemple de Denjoy analytiques, C. R. Acad. Sci. Paris Ser. I. Math. 289 (1984), 141-144.

[Y2] _ Manuscript, 1990.

Department of Mathematics and Institute for Mathematical Sciences, State University of New York at Stony Brook, Stony Brook, New York 11794

E-mail address, M. Lyubich: mlyubich@math.sunysb.edu

E-mail address, J. Milnor: jack@math.sunysb.edu 